Journal of Applied Pharmaceutical Science Vol. 7 (05), pp. 204-218, May, 2017

Available online at http://www.japsonline.com

DOI: 10.7324/JAPS.2017.70534

ISSN 2231-3354 (cc) BY-NC-sA

\title{
Phytochemistry and Biological Activities of the Genus Ocotea (Lauraceae): A Review on Recent Research Results (2000-2016)
}

\author{
Wan Mohd Nuzul Hakimi Wan Salleh ${ }^{1 *}$, Farediah Ahmad ${ }^{2}$ \\ ${ }^{1}$ Department of Chemistry, Faculty of Science and Mathematics, Universiti Pendidikan Sultan Idris (UPSI), 35900 Tanjong Malim, Perak, Malaysia. \\ ${ }^{2}$ Department of Chemistry, Faculty of Science, Universiti Teknologi Malaysia (UTM), 81310 Johor Bahru, Johor, Malaysia.
}

\begin{tabular}{|c|c|}
\hline ARTICLE INFO & ABSTRACT \\
\hline $\begin{array}{l}\text { Article history: } \\
\text { Received on: } 29 / 12 / 2016 \\
\text { Accepted on: } 11 / 02 / 2017 \\
\text { Available online: } 30 / 05 / 2017\end{array}$ & $\begin{array}{l}\text { Ocotea (family: Lauracea), which comprises nearly } 350 \text { species, are distributed throughout tropical America, } \\
\text { Africa, and Asia. Up to now, the reported constituents from the genus Ocotea involve neolignans, alkaloids, } \\
\text { sesquiterpenes, flavonoids, lignans, butanolides, benzopyrans, steroids, essential oils and several other types of } \\
\text { compounds (alkylphenols, arylpropene, coumarin, ester, saponin). Studies have shown that Ocotea and its active }\end{array}$ \\
\hline $\begin{array}{l}\text { Key words: } \\
\text { Phytochemistry; } \\
\text { pharmacology; Ocotea; } \\
\text { Lauraceae. }\end{array}$ & $\begin{array}{l}\text { principles possess a wide range of pharmacological activities, such as anti-nflammatory, cytotoxicity, } \\
\text { antimicrobial, larvicidal, and antiproliferative activities. The outcome of these studies will further support the } \\
\text { therapeutic potential of the genus Ocotea, and provide convincing evidences to its future clinical applications in } \\
\text { modern medicine. Thus, increasing amount of data supports application and exploitation for new drug } \\
\text { development. }\end{array}$ \\
\hline
\end{tabular}

\section{INTRODUCTION}

The plant genus Ocotea, one of the largest members of the Lauraceae family, comprises approximately 350 species that are distributed throughout tropical and subtropical climates. Most species are found in America from Mexico to Argentina, seven species are found in Africa, one species is found in the Canary Islands, and about 34 recognized species are found in Madagascar (Rohwer, 2000; van der Werff, 2013). It can be recognized by the simple, alternate, stiff and aromatic elliptic to obovate leaves and fruits often borne in a cup. This family has a considerable economic importance worldwide because it is used as a source of timber for construction and furniture (Nectandra, Ocotea, Persea spp.), as a crop (Persea americana), and to obtain flavours for food industry, perfumery, and medicines (Cinnamomum zeylanicum, C. cassia) (Chaverri et al., 2011). Several plants of this genus have been used for the treatment of various diseases. Among them, the stem wood of $O$. bullata has been used to treat headache and male urinary tract infections

* Corresponding Author

Email: wmnhakimi @ fsmt.upsi.edu.my
(Rakotondraibe et al., 2015). O. puchury-major is popular in local medicine, as possible sedative, gastroenteric, and antirheumatic properties. It is reported mainly for its leaves and bark, as well as cosmetic applications involving the essential oil of the leaves (Christophel et al., 1996). O. quixos is used as disinfectant, local anaesthetic and anti-diarrheic infusion (Ballabeni et al., 2007). Meanwhile, $O$. lancifolia is used as antiparasitic, and $O$. caparrapi is used to treat insect bites, snake bites, bronchitis, and cancerous tumours (Fournet et al., 2007). The woody calyces of $O$. bofo collected from mature fruits are traditionally used to aromatize infusions by ethnic groups. It possesses a strong anise like aroma and thus may represents a potential aniseed, fennel, or tarragon substitute or adulterant (Guerrini et al., 2006). A number of plants in the genus Ocotea are the sources of secondary metabolites, including neolignans, alkaloids, flavonoids, sesquiterpenes, lignans, butanolides, benzopyrans, steroids, alkylphenols, arylpropenes, coumarins, esters, and saponins; many of which exhibited interesting antiproliferative, antifungal, antiherpetic, antiinflammatory, and antimicrobial activities (Camargo et al., 2013; Castro et al., 2011; Cuca et al., 2009; Destryana et al., 2014; Garcez et al., 2011; Garett et al., 2012; Yamaguchi et al., 2013). 
The extensive reading and investigation were actualized by systematically searching the scientific databases (PubMed, Scopus, SciFinder, and the Web of Science) for topics related to factors like the essential oils composition, chemical constituents, and pharmacological effects of the genus Ocotea. A bibliographic search, carried out from the year 2000 to 2016 of the genus Ocotea revealed that about 43 species were investigated at chemical or biological level.

Due to the ethnobotanic importance of this genus, further studies on Ocotea species are urgently needed. Thus, the aim of this review is to provide an overview on chemical and pharmacological studies on the essential oil, extracts, and isolated compounds from the genus Ocotea from year 2000 to 2016. Also included are the biological activities of compounds isolated in recent years. This should be helpful for professionals in ethnopharmacology and natural product chemistry and promote the application of plants of this genus.

\section{PHYTOCHEMISTRY STUDIES}

The chemical constituents of Ocotea compounds (Figure 1) includeneolignans, alkaloids, sesquiterpenes, flavonoids lignans, butanolides, benzopyrans, steroids, alkylphenols, arylpropene, coumarins, ester, and saponin. Their structures are shown below, and their names and the corresponding plant sources are listed in the Table 1. In addition, the chemical compositions of the Ocotea essential oils are also discussed and summarized in Table 2.

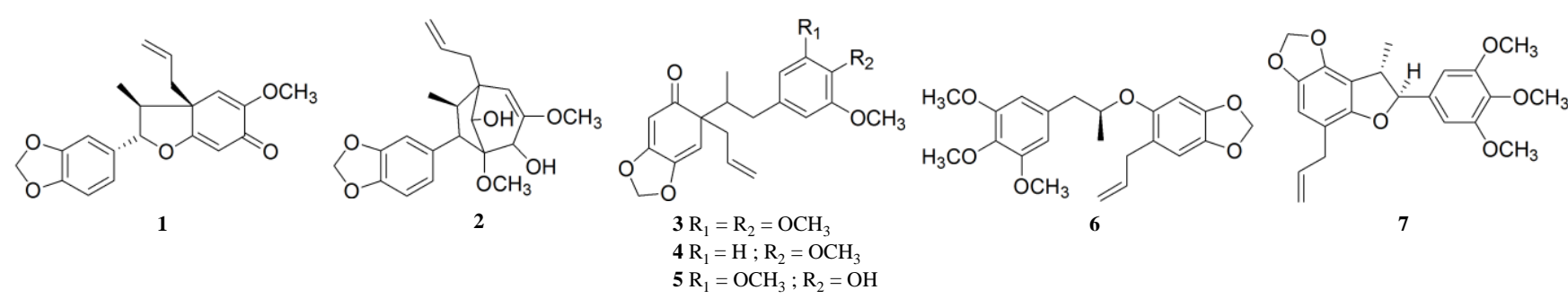

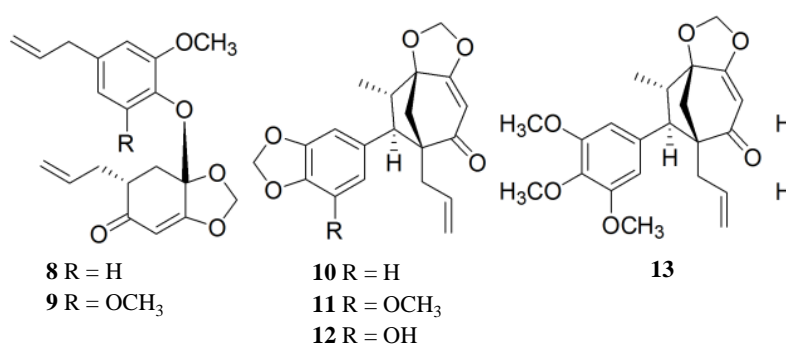<smiles>C=CCC1C(=O)C=C2OCOC2[C@H](C)[C@@H]1c1cc(OC)c(OC)c(OC)c1</smiles><smiles>[R]c1cc(CC=C)cc(OC)c1OC(C)Cc1cc(OC)c(OC)c(OC)c1</smiles><smiles>C=CCc1cc(OC)c(OC(C)Cc2cc(OC)c(OC)c(OC)c2)c(OC)c1</smiles>

$15 \mathrm{R}=\mathrm{OCH}_{3}$ 17<smiles>C=CCC1C(=O)C=C2OCOC2[C@H](C)[C@@H]1c1cc(OC)c2c(c1)OCO2</smiles><smiles>[R]c1cc([C@@H]2[C@@H]3O[C@](C)(C=C(CC=C)C3=O)[C@@H]2OC)cc([R])c1[R]</smiles>

$$
20 \mathrm{R}_{1}=\mathrm{R}_{2}=\mathrm{OCH}_{2} \mathrm{O} ; \mathrm{R}_{3}=\mathrm{H}
$$
$21 \mathrm{R}_{1}=\mathrm{R}_{2}=\mathrm{R}_{3}=\mathrm{OCH}_{3}$

$$
\begin{aligned}
& 22 \mathrm{R}_{1}=\mathrm{R}_{2}=\mathrm{OCH}_{2} \mathrm{O} ; \mathrm{R}_{3}=\mathrm{H} \\
& \mathbf{2 3} \mathrm{R}_{1}=\mathrm{R}_{2}=\mathrm{OCH}_{3} ; \mathrm{R}_{3}=\mathrm{H}
\end{aligned}
$$
$24 \mathrm{R}_{1}=\mathrm{R}_{2}=\mathrm{R}_{3}=\mathrm{OCH}_{3}$

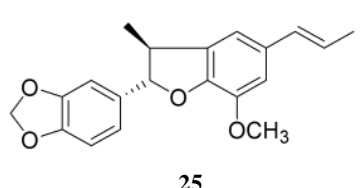

25<smiles></smiles>

26<smiles>CC(Cc1ccc(O)c(O)c1)C(C)Cc1ccc(O)c(O)c1</smiles>

27<smiles>[R]c1cc([C@@H]2O[C@]3(O)C(=O)C=CC[C@]3(CC=C)[C@H]2C)cc([R3])c1[R]</smiles>

$28 \mathrm{R}_{1}=\mathrm{R}_{2}=\mathrm{OCH}_{2} \mathrm{O} ; \mathrm{R}_{3}=\mathrm{H}$ $29 \mathrm{R}_{1}=\mathrm{R}_{2}=\mathrm{OCH}_{3} ; \mathrm{R}_{3}=\mathrm{H}$ $30 \mathrm{R}_{1}=\mathrm{R}_{2}=\mathrm{R}_{3}=\mathrm{OCH}_{3}$
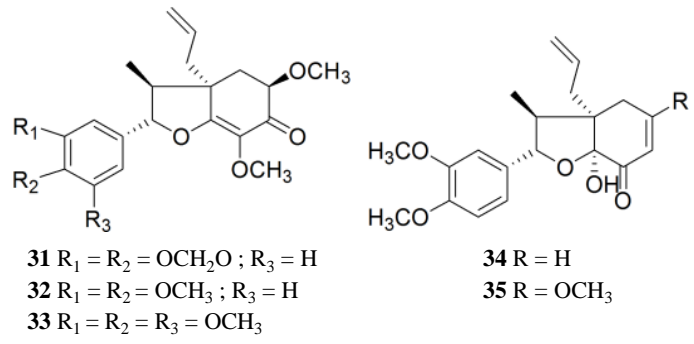


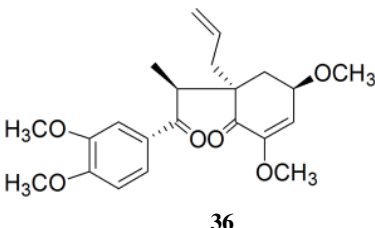

36<smiles>[R]c1cc2c(cc1[R4])-c1c([R])c([R])c([R3])c3c1C(C2)N([R6])CC3</smiles>

$42 \mathrm{R}_{1}=\mathrm{R}_{2}=\mathrm{OCH}_{2} \mathrm{O} ; \mathrm{R}_{3}=\mathrm{H} ; \mathrm{R}_{4}=\mathrm{R}_{5}=\mathrm{OCH}_{3} ; \mathrm{R}_{6}=\mathrm{CH}_{3} ;(6 \mathrm{aS})$ $48 \mathrm{R}_{1}=\mathrm{R}_{2}=\mathrm{OCH}_{2} \mathrm{O} ; \mathrm{R}_{3}=\mathrm{H} ; \mathrm{R}_{4}=\mathrm{R}_{5}=\mathrm{OCH}_{2} \mathrm{O} ; \mathrm{R}_{6}=\mathrm{CH}_{3} ;(6 \mathrm{aS})$ $53 \mathrm{R}_{1}=\mathrm{R}_{2}=\mathrm{OCH}_{3} ; \mathrm{R}_{3}=\mathrm{H} ; \mathrm{R}_{4}=\mathrm{R}_{5}=\mathrm{OCH}_{2} \mathrm{O} ; \mathrm{R}_{6}=\mathrm{CH}_{3} ;(6 \mathrm{a}-7)$ $54 \mathrm{R}_{1}=\mathrm{R}_{2}=\mathrm{OCH}_{3} ; \mathrm{R}_{3}=\mathrm{H} ; \mathrm{R}_{4}=\mathrm{R}_{5}=\mathrm{OCH}_{2} \mathrm{O} ; \mathrm{R}_{6}=\mathrm{CH}_{3} ;(6 \mathrm{aS})$ $55 \mathrm{R}_{1}=\mathrm{R}_{2}=\mathrm{OCH}_{3} ; \mathrm{R}_{3}=\mathrm{H} ; \mathrm{R} 4=\mathrm{R}_{5}=\mathrm{OCH}_{2} \mathrm{O} ; \mathrm{R}_{6}=\mathrm{COCH}_{3} ;(6 \mathrm{aS})$ $56 \mathrm{R}_{1}=\mathrm{R}_{2}=\mathrm{OCH}_{2} \mathrm{O} ; \mathrm{R}_{3}=\mathrm{OCH}_{3} ; \mathrm{R}_{4}=\mathrm{R}_{5}=\mathrm{OCH}_{2} \mathrm{O} ; \mathrm{R}_{6}=\mathrm{H} ;(6 \mathrm{aS})$ $57 \mathrm{R}_{1}=\mathrm{R}_{2}=\mathrm{OCH}_{2} \mathrm{O} ; \mathrm{R}_{3}=\mathrm{OCH}_{3} ; \mathrm{R}_{4}=\mathrm{R}_{5}=\mathrm{OCH}_{3} ; \mathrm{R}_{6}=\mathrm{CH}_{3} ;(6 \mathrm{a}-7)$

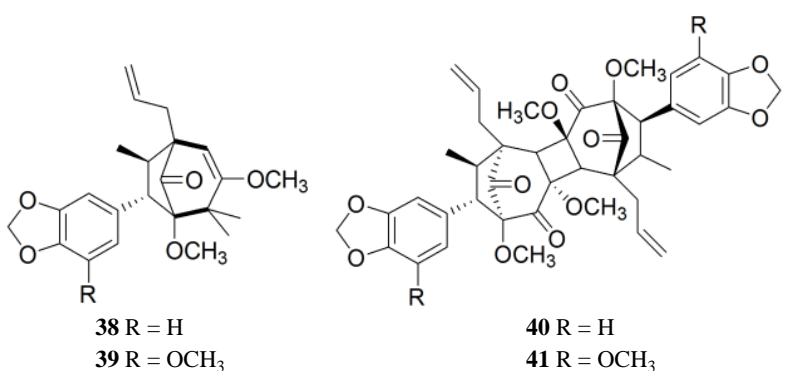

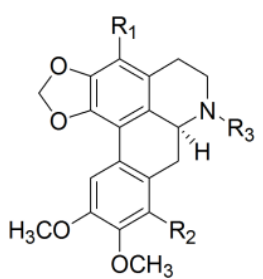

$43 \mathrm{R}_{1}=\mathrm{OCH}_{3} ; \mathrm{R}_{2}=\mathrm{H} ; \mathrm{R}_{3}=\mathrm{CH}_{3}$ $45 \mathrm{R}_{1}=\mathrm{H} ; \mathrm{R}_{2}=\mathrm{OH} ; \mathrm{R}_{3}=\mathrm{CH}_{3}$ $46 \mathrm{R}_{1}=\mathrm{OCH}_{3} ; \mathrm{R}_{2}=\mathrm{OH} ; \mathrm{R}_{3}=\mathrm{H}$

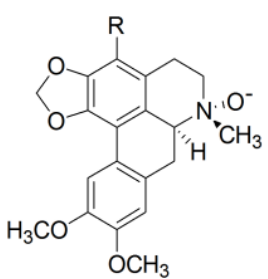

$44 \mathrm{R}=\mathrm{OCH}_{3}$ $72 \mathrm{R}=\mathrm{H}$

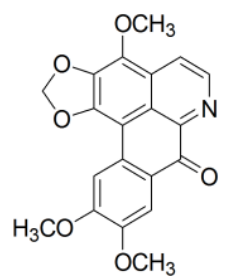

47

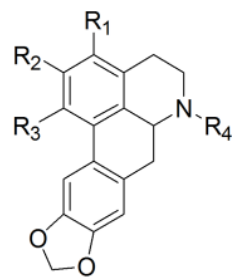

$49 \mathrm{R}_{1}=\mathrm{R}_{2}=\mathrm{OCH}_{3} ; \mathrm{R}_{3}=\mathrm{OH} ; \mathrm{R}_{4}=\mathrm{H}$

$50 \mathrm{R}_{1}=\mathrm{R}_{2}=\mathrm{OCH}_{3} ; \mathrm{R}_{3}=\mathrm{OH} ; \mathrm{R}_{4}=\mathrm{COOCH}_{2} \mathrm{CH}_{3}$

$51 \mathrm{R}_{1}=\mathrm{R}_{2}=\mathrm{OCH}_{3} ; \mathrm{R}_{3}=\mathrm{OH} ; \mathrm{R}_{4}=\mathrm{COH}$

$52 \mathrm{R}_{1}=\mathrm{R}_{2}=\mathrm{OCH}_{3} ; \mathrm{R}_{3}=\mathrm{OH} ; \mathrm{R}_{4}=\mathrm{COOCH}_{3}$

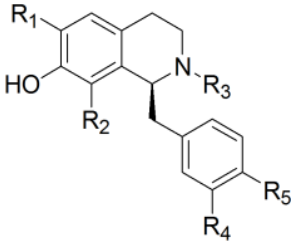

$58 \mathrm{R}_{1}=\mathrm{OCH}_{3} ; \mathrm{R}_{2}=\mathrm{R}_{3}=\mathrm{R}_{4}=\mathrm{H} ; \mathrm{R}_{5}=\mathrm{OH}$

$59 \mathrm{R}_{1}=\mathrm{OCH}_{3} ; \mathrm{R}_{2}=\mathrm{R}_{4}=\mathrm{H} ; \mathrm{R}_{3}=\mathrm{CH}_{3} ; \mathrm{R}_{5}=\mathrm{OH}$

$69 \mathrm{R}_{1}=\mathrm{R}_{3}=\mathrm{R}_{4}=\mathrm{H} ; \mathrm{R}_{2}=\mathrm{OCH}_{3} ; \mathrm{R}_{3}=\mathrm{H} ; \mathrm{R}_{5}=\mathrm{OH}$

$70 \mathrm{R}_{1}=\mathrm{R}_{5}=\mathrm{OCH}_{3} ; \mathrm{R}_{2}=\mathrm{H} ; \mathrm{R}_{3}=\mathrm{CH}_{3} ; \mathrm{R}_{4}=\mathrm{OH}$

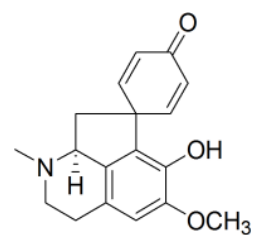

60<smiles>COc1cc2c3c(c1O)-c1ccccc1CNC3CC2</smiles>

61

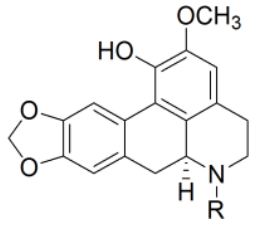

$63 \mathrm{R}=\mathrm{H}$ $66 \mathrm{R}=\mathrm{CH}_{3}$

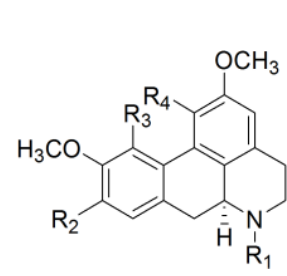

62 $\mathrm{R}_{1}=\mathrm{R}_{3}=\mathrm{H} ; \mathrm{R}_{2}=\mathrm{OH} ; \mathrm{R}_{4}=\mathrm{OCH}_{3}$

$64 \mathrm{R}_{1}=\mathrm{R}_{3}=\mathrm{H} ; \mathrm{R}_{2}=\mathrm{R}_{4}=\mathrm{OH}$

$65 \mathrm{R}_{1}=\mathrm{CH}_{3} ; \mathrm{R}_{2}=\mathrm{H} ; \mathrm{R}_{3}=\mathrm{R}_{4}=\mathrm{OH}$

$67 \mathrm{R}_{1}=\mathrm{CH}_{3} ; \mathrm{R}_{2}=\mathrm{R}_{4}=\mathrm{OH} ; \mathrm{R}_{3}=\mathrm{H}$<smiles></smiles>

$73 \mathrm{R}_{1}=\mathrm{CH}_{3} ; \mathrm{R}_{2}=\mathrm{R}_{5}=\mathrm{R}_{6}=\mathrm{OCH}_{3} ; \mathrm{R}_{3}=\mathrm{R}_{4}=\mathrm{OCH}_{2} \mathrm{O} ; \mathrm{R}_{7}=\mathrm{OH}$

$74 \mathrm{R}_{1}=\mathrm{R}_{7}=\mathrm{H} ; \mathrm{R}_{2}=\mathrm{R}_{5}=\mathrm{R}_{6}=\mathrm{OCH}_{3} ; \mathrm{R}_{3}=\mathrm{R}_{4}=\mathrm{OCH}_{2} \mathrm{O}$ $75 \mathrm{R}_{1}=\mathrm{R}_{2}=\mathrm{R}_{7}=\mathrm{H} ; \mathrm{R}_{3}=\mathrm{R}_{4}=\mathrm{OCH} 2 \mathrm{O} ; \mathrm{R}_{5}=\mathrm{R}_{6}=\mathrm{OCH}_{3}$ $76 \mathrm{R}_{1}=\mathrm{CH}_{3} ; \mathrm{R}_{2}=\mathrm{R}_{3}=\mathrm{R}_{4}=\mathrm{R}_{5}=\mathrm{R}_{6}=\mathrm{OCH}_{3} ; \mathrm{R}_{7}=\mathrm{H}$ $77 \mathrm{R}_{1}=\mathrm{R}_{2}=\mathrm{R}_{7}=\mathrm{H} ; \mathrm{R}_{3}=\mathrm{OH} ; \mathrm{R}_{4}=\mathrm{OCH}_{3} ; \mathrm{R}_{5}=\mathrm{R}_{6}=\mathrm{OCH}_{2} \mathrm{O}$ $78 \mathrm{R}_{1}=\mathrm{CH}_{3} ; \mathrm{R}_{2}=\mathrm{R}_{7}=\mathrm{H} ; \mathrm{R}_{3}=\mathrm{OH} ; \mathrm{R}_{4}=\mathrm{R}_{5}=\mathrm{R}_{6}=\mathrm{OCH}_{3}$ $79 \mathrm{R}_{1}=\mathrm{CH}_{3} ; \mathrm{R}_{2}=\mathrm{R}_{7}=\mathrm{H} ; \mathrm{R}_{3}=\mathrm{R}_{4}=\mathrm{R}_{5}=\mathrm{OCH}_{3} ; \mathrm{R}_{6}=\mathrm{OH}$<smiles>OCCc1c[nH]c2ccc(OC3(O)OCC(O)C(O)C(O)C3O)cc12</smiles>

71<smiles>Oc1ccc(C2Oc3c(O)cc(O)c4c3OC(c3ccc(O)c(O)c3)C(c3c(O)cc(O)c5c3OC(c3ccc(O)c(O)c3)(C4O)C5O)C2O)c(O)c1</smiles>

80<smiles>[R2]Oc1c(-c2ccc(O)c([R2])c2)oc2cc(O)cc(O)c2c1=O</smiles>

$\mathbf{8 1} \mathrm{R}_{1}=$ Glucose $; \mathrm{R}_{2}=\mathrm{OH}$

$82 \mathrm{R}_{1}=$ Xylose $; \mathrm{R}_{2}=\mathrm{OH}$

$83 \mathrm{R}_{1}=$ Glucuronic acid $; \mathrm{R}_{2}=\mathrm{OH}$

$84 \mathrm{R}_{1}=$ Rhamnose $; \mathrm{R}_{2}=\mathrm{OH}$

$85 \mathrm{R}_{1}=$ Rhamnose $; \mathrm{R}_{2}=\mathrm{H}$

$88 \mathrm{R}_{1}=\mathrm{H} ; \mathrm{R}_{2}=\mathrm{OH}$

$89 \mathrm{R}_{1}=\mathrm{R}_{2}=\mathrm{H}$<smiles>[R2]C1Cc2c(O)cc(O)cc2OC1c1ccc(O)c(O)c1</smiles>

$86 \mathrm{R}_{1}=\mathrm{OH} ; \mathrm{R}_{2}=\mathrm{H}$

$87 \mathrm{R}_{1}=\mathrm{H} ; \mathrm{R}_{2}=\mathrm{OH}$ 


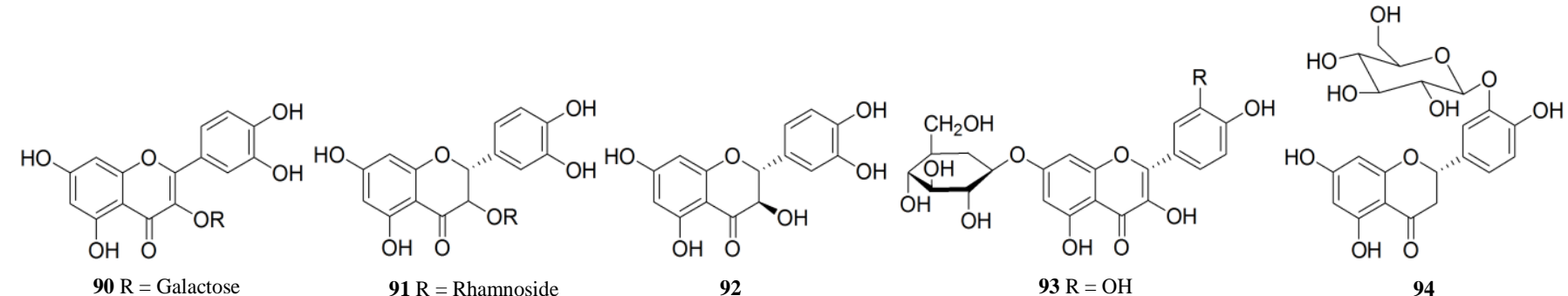

$90 \mathrm{R}=$ Galactose

$91 \mathrm{R}=$ Rhamnoside

$93 \mathrm{R}=\mathrm{OH}$
$95 \mathrm{R}=\mathrm{H}$

94

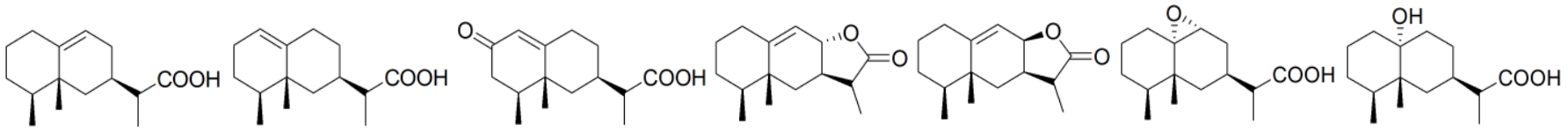

96

97

98

99

100

101

102

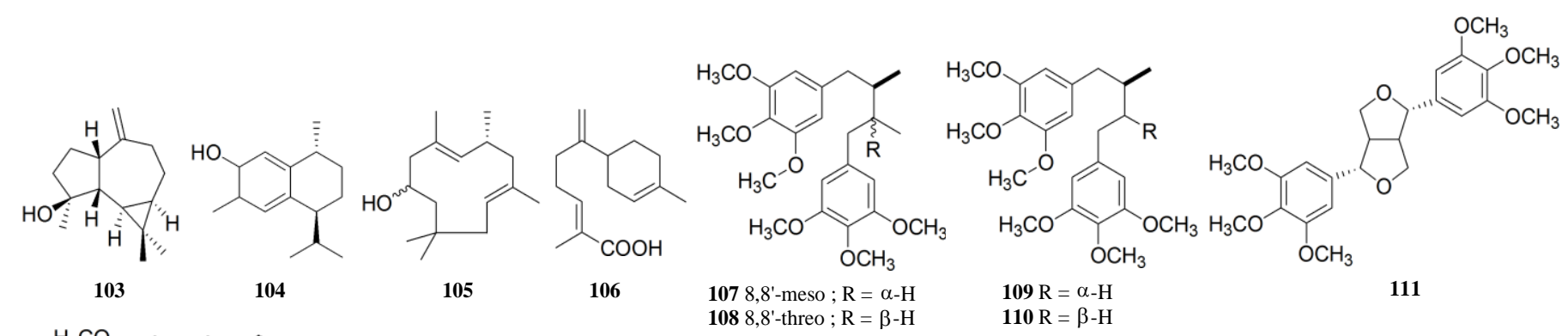<smiles>COc1cc([C@H]2c3c(cc(OC)c(O)c3OC)C[C@H](CO)[C@H]2CO)cc(OC)c1O</smiles><smiles>C=C(C)C=C1C(=O)OC(=C)[C@@H]1O</smiles>

$1133 E, 4 S ; \mathrm{n}=12 \quad 1142 E, 1^{\prime} S ; \mathrm{n}=14$ $1163 Z, 4 R ; \mathrm{n}=11 \quad 1152 E, 1 ' S ; \mathrm{n}=12$<smiles>COc1cccc2oc(C)cc(=O)c12</smiles>

118<smiles>COc1cccc2oc(C)cc(=O)c12</smiles>

120

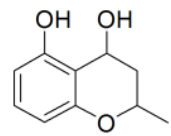

121<smiles>C=C[C@H](C)[C@H]1CC[C@@H]2[C@@]1(C)CC[C@@H]1[C@@]3(C)CCC(=O)C=C3CC[C@@]12C</smiles>

122

123

124

125

126

127

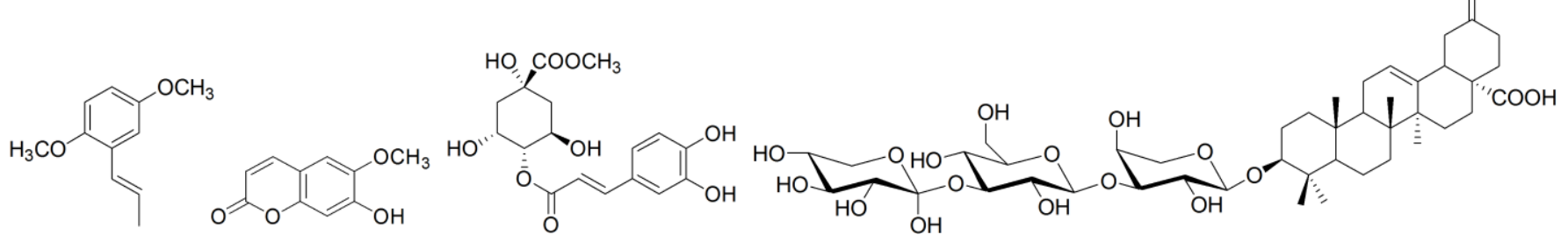

128

129

130

131

Fig. 1: Chemical structures of the compounds isolated from the genus Ocotea. 
Table 1: Chemical constituents isolated from the genus Ocotea (2000-2016).

\begin{tabular}{|c|c|c|c|}
\hline No. & Compound class and name & Source & References \\
\hline & Neolignans & & \\
\hline \multirow[t]{2}{*}{1} & Burchellin & O. cymbarum & Narciso et al., 2014 \\
\hline & & O. elegans & Oliveira et al., 2006 \\
\hline 2 & Canelin & O. elegans & Oliveira et al., 2006 \\
\hline 3 & Cymosalignan A & O. cymosa & Rakotondraibe et al., 2015 \\
\hline 4 & Cymosalignan B & O. cymosa & Rakotondraibe et al., 2015 \\
\hline 5 & Cymosalignan $\mathrm{C}$ & O. cymosa & Rakotondraibe et al., 2015 \\
\hline 6 & $3^{\prime}, 4^{\prime}$-Methylenedioxy-3,4,5-trimethoxy- $\Delta^{8^{\prime}}-8.0 .6^{\prime}$-neolignan & O. cymosa & Rakotondraibe et al., 2015 \\
\hline 7 & Ococymosin & O. cymosa & Rakotondraibe et al., 2015 \\
\hline 8 & Didymochlaenone B & O. суmosa & Rakotondraibe et al., 2015 \\
\hline 9 & Didymochlaenone C & O. cymosa & Rakotondraibe et al., 2015 \\
\hline 10 & Sibyllenone & O. bullata & Zschocke et al., 2000 \\
\hline 11 & Demethoxysibyllenone & O. cymosa & Rakotondraibe et al., 2015 \\
\hline 12 & 5-O-Demethylsibyllenone & O. cymosa & Rakotondraibe et al., 2015 \\
\hline 13 & $\begin{array}{l}\left(7 R, 8 S, 1^{\prime} S, 3^{\prime} S\right) \text { - } \Delta^{8^{\prime}}-3,4,5 \text {-Trimethoxy-3', } 4^{\prime} \text {-methylenedioxy-1', } 2^{\prime}, 3^{\prime}, 6^{\prime} \text {-tetrahydro- } \\
6^{\prime} \text {-oxo-7.1'-8. }\end{array}$ & O. cymosa & Rakotondraibe et al., 2015 \\
\hline 14 & $\begin{array}{l}\left(7 R, 8 R, 1^{\prime} R, 3^{\prime} R\right)-\Delta^{8^{\prime}}-3,4,5 \text {-Trimethoxy-3', } 4^{\prime} \text {-methylenedioxy-1' }, 2^{\prime}, 3^{\prime}, 6^{\prime} \text {-tetrahydro- } \\
6^{\prime} \text {-oxo-7.1'-8.3'-neolignan }\end{array}$ & O. cymosa & Rakotondraibe et al., 2015 \\
\hline 15 & $3,4,5,3^{\prime}, 5^{\prime}$-Pentamethoxy-1'-allyl-8.O.4'-neolignan & O. cymosa & Rakotondraibe et al., 2015 \\
\hline 16 & 3,4,5,3'-Tetramethoxy-1'-allyl-8.O.4'-neolignan & O. суmosa & Rakotondraibe et al., 2015 \\
\hline 17 & Virolongin B & O. сутова & Rakotondraibe et al., 2015 \\
\hline \multirow[t]{2}{*}{18} & Ocobullenone & O. cymosa & Rakotondraibe et al., 2015 \\
\hline & & O. bullata & Zschocke et al., 2000 \\
\hline \multirow[t]{2}{*}{19} & 2'-Epiguianin & O. macrophylla & Suárez et al., 2011 \\
\hline & & O. macrophylla & Coy-Barerra et al., 2009 \\
\hline \multirow[t]{2}{*}{20} & Ocophyllal A & O. macrophylla & Coy-Barerra et al., 2009 \\
\hline & & O. macrophylla & Suárez et al., 2011 \\
\hline \multirow[t]{2}{*}{21} & Ocophyllal B & O. macrophylla & Coy-Barerra et al., 2009 \\
\hline & & O. macrophylla & Suárez et al., 2011 \\
\hline \multirow[t]{2}{*}{22} & Ocophyllol A & O. macrophylla & Coy-Barerra et al., 2009 \\
\hline & & O. macrophylla & Suárez et al., 2011 \\
\hline \multirow[t]{2}{*}{23} & Ocophyllol B & O. macrophylla & Coy-Barerra et al., 2009 \\
\hline & & O. macrophylla & Suárez et al., 2011 \\
\hline 24 & Ocophyllol C & O. macrophylla & Coy-Barerra et al., 2009 \\
\hline 25 & $(+)$-Licarin B & O. macrophylla & Coy-Barerra et al., 2009 \\
\hline 26 & $\begin{array}{l}\text { rel- }\left(7 S, 8 R, 1^{\prime} S, 2^{\prime} R, 3^{\prime} S\right)-\Delta^{8^{\prime}}-2^{\prime} \text {-hydroxy-5,1',3'-trimethoxy-3,4-methylenedioxy-7,3', } \\
8,1^{\prime} \text {-neolignan }\end{array}$ & O. heterochroma & Cuca et al., 2009 \\
\hline 27 & meso-Dehydroguaiaretic acid & O. heterochroma & Cuca et al., 2009 \\
\hline 28 & Ferrearin $\mathrm{C}$ & O. catharinensis & Funasaki et al., 2009 \\
\hline 29 & Ferrearin E & O. catharinensis & Funasaki et al., 2009 \\
\hline 30 & Ferrearin G & O. catharinensis & Funasaki et al., 2009 \\
\hline 31 & Armenin B & O. catharinensis & Funasaki et al., 2009 \\
\hline 32 & 5'-Methoxyporosin & O. catharinensis & Funasaki et al., 2009 \\
\hline 33 & $\left(7 S, 8 S, 1^{\prime} R, 3^{\prime} R\right)-3,4,5,3^{\prime}, 5^{\prime}$-Pentamethoxy-4'-oxo- $\Delta^{1,3,5,5^{\prime}, 8^{\prime}}-8.1^{\prime}, 7.0 .6^{\prime}$-neolignan & O. catharinensis & Funasaki et al., 2009 \\
\hline 34 & $\begin{array}{l}r e l- \\
\text { neolignan }\end{array} \quad\left(7 S, 8 S, 1^{\prime} R, 2^{\prime} S\right)-2^{\prime}$-Hydroxy-3,4-dimethoxy-3'-oxo- $\Delta^{1,3,5,4^{\prime}, 8^{\prime}}-8.1^{\prime}, 7.0 .2^{\prime}-$ & O. catharinensis & Funasaki et al., 2009 \\
\hline 35 & $\begin{array}{l}\text { rel- }\left(7 R, 8 S, 1^{\prime} R, 2^{\prime} S\right)-2^{\prime} \text {-Hydroxy-3,4,5'-trimethoxy-3'-oxo- } \Delta^{1,3,5,4^{\prime}, 8^{\prime}}-8.1^{\prime}, 7.0 .2^{\prime}- \\
\text { neolignan }\end{array}$ & O. catharinensis & Funasaki et al., 2009 \\
\hline 36 & rel- $\left(8 S, 1^{\prime} R, 5^{\prime} R\right)-3,4,3^{\prime}, 5^{\prime}-$ Tetramethoxy-7, $2^{\prime}$-dioxo- $\Delta^{1,3,5,3^{\prime}, 8^{\prime}}-8.1^{\prime}$-neolignan & O. catharinensis & Funasaki et al., 2009 \\
\hline 37 & $\begin{array}{l}\text { rel- } \quad\left(7 R, 8 S, 1^{\prime} R, 2^{\prime} S\right)-2^{\prime} \text {-Hydroxy-3,4-methylenedioxy-5, } 3^{\prime}, 5^{\prime} \text {-trimethoxy- } \Delta^{1,3,5,3^{\prime}, 8^{\prime}} \text { - } \\
8.1^{\prime}, 7 . O .2^{\prime} \text {-neolignan }\end{array}$ & O. catharinensis & Funasaki et al., 2009 \\
\hline 38 & $\begin{array}{l}r e l-\left(7 S, 8 R, 1^{\prime} R, 3^{\prime} R\right)-4^{\prime}-\text { Hydroxy-3,4-methylenedioxy-3', } 5^{\prime} \text {-dimethoxy-2', } 4^{\prime} \text {-dioxo- } \\
\Delta^{1,3,5,5, S^{\prime}}-8.1^{\prime}, 7.3^{\prime} \text {-neolignan }\end{array}$ & O. catharinensis & Funasaki et al., 2009 \\
\hline 39 & $\begin{array}{l}\left(7 S, 8 R, 1^{\prime} R, 3^{\prime} R\right)-4^{\prime} \text {-Hydroxy-3,4-methylenedioxy-3',5',5-trimethoxy-2', } 4^{\prime} \text {-dioxo- } \\
\Delta^{1,3,5,5^{\prime}, 8^{\prime}}-8.1^{\prime}, 7.3^{\prime} \text {-neolignan }\end{array}$ & O. catharinensis & Funasaki et al., 2009 \\
\hline 40 & $\begin{array}{l}r e l-\left(7 S, 8 R, 1^{\prime} R, 3^{\prime} R\right)-4^{\prime}-\text { Hydroxy-3,4-methylenedioxy-3', } 5^{\prime} \text {-dimethoxy-2', } 4^{\prime} \text {-dioxo- } \\
\Delta^{1,3,5,5 S^{\prime}, 8^{\prime}}-8.1^{\prime}, 7.3^{\prime} \text {-neolignan dimer }\end{array}$ & O. catharinensis & Funasaki et al., 2009 \\
\hline \multirow[t]{2}{*}{41} & $\begin{array}{l}\left(7 S, 8 R, 1^{\prime} R, 3^{\prime} R\right)-4^{\prime} \text {-Hydroxy-3,4-methylenedioxy-3', } 5^{\prime}, 5 \text {-trimethoxy-2', } 4^{\prime} \text {-dioxo- } \\
\Delta^{1,3,5,5^{\prime}, 8^{\prime}}-8.1^{\prime}, 7.3^{\prime} \text {-neolignan dimer }\end{array}$ & O. catharinensis & Funasaki et al., 2009 \\
\hline & Alkaloids & & \\
\hline \multirow{3}{*}{42} & (+)-Dicentrine & O. puberula & Montrucchio et al., 2012 \\
\hline & & O. macrophylla & Coy-Barrera and Cuca-Suárez, 2009 \\
\hline & & O. acutifolia & Garcez et al., 2011; Guterres et al., 2013 \\
\hline 43 & $(+)$-Ocoteine & O. acutifolia & Garcez et al., 2011; Guterres et al., 2013 \\
\hline 44 & (+)-6S-Ocoteine $N$-oxide & O. acutifolia & Garcez et al., 2011; Guterres et al., 2013 \\
\hline 45 & $(+)$-Leucoxine & O. acutifolia & Garcez et al., 2011; Guterres et al., 2013 \\
\hline
\end{tabular}




\begin{tabular}{|c|c|c|c|}
\hline 46 & (+)-Norocoxylonine & O. acutifolia & Garcez et al., 2011 \\
\hline 47 & (+)-Thalicminine & O. acutifolia & Garcez et al., 2011; Guterres et al., 2013 \\
\hline \multirow[t]{2}{*}{48} & $(+)$-Neolitsine & O. acutifolia & Garcez et al., 2011; Guterres et al., 2013 \\
\hline & & O. macrophylla & Coy-Barrera and Cuca-Suárez 2009 \\
\hline 49 & $S$-3-methoxy-nordomesticine & O. macrophylla & Pabon and Cuca 2010 \\
\hline 50 & $S$ - $N$-ethoxycarbonyl-3-methoxy-nordomesticine & O. macrophylla & Pabon and Cuca 2010 \\
\hline 51 & $S$ - $N$-formyl-3-methoxy-nordomesticine & O. macrophylla & Pabon and Cuca 2010 \\
\hline 52 & $S$ - $N$-methoxycarbonyl-3-methoxy-nordomesticine & O. macrophylla & Pabon and Cuca 2010 \\
\hline 53 & Dehydronantenine & O. macrophylla & Coy-Barrera and Cuca-Suárez 2009 \\
\hline 54 & (+)-Nantenine & O. macrophylla & Coy-Barrera and Cuca-Suárez 2009 \\
\hline 55 & (+)-N-acetyl-nornantenine & O. macrophylla & Coy-Barrera and Cuca-Suárez 2009 \\
\hline 56 & (+)-Cassythidine & O. macrophylla & Coy-Barrera and Cuca-Suárez 2009 \\
\hline 57 & Didehydroocoteine & O. macrophylla & Coy-Barrera and Cuca-Suárez 2009 \\
\hline \multirow[t]{2}{*}{58} & Coclaurine & O. lancifolia & Fournet et al., 2007 \\
\hline & & O. duckei & Silva et al., 2002 \\
\hline 59 & $(-)-N$-Methycoclaurine & O. lancifolia & Fournet et al., 2007 \\
\hline NMS & Crostparine & O. lancifolia & Fournet et al., 2007 \\
\hline 60 & Glaziovine & O. lancifolia & Fournet et al., 2007 \\
\hline 61 & (-)-Caaverine & O. lancifolia & Fournet et al., 2007 \\
\hline 62 & (+)-Laurotetanine & O. lancifolia & Fournet et al., 2007 \\
\hline 63 & (+)-Nordomesticine & O. lancifolia & Fournet et al., 2007 \\
\hline 64 & (+)-Norisoboldine & O. lancifolia & Fournet et al., 2007 \\
\hline NMS & (+)-Norantenine & O. lancifolia & Fournet et al., 2007 \\
\hline 65 & $(+)$-Corytuberine & O. lancifolia & Fournet et al., 2007 \\
\hline 66 & (+)-Domesticine & O. lancifolia & Fournet et al., 2007 \\
\hline 67 & (+)-Isoboldine & O. lancifolia & Fournet et al., 2007 \\
\hline 68 & $(S)$-Pallidine & O. lancifolia & Fournet et al., 2007 \\
\hline 69 & (+)-Norjuziphine & O. lancifolia & Fournet et al., 2000 \\
\hline 70 & (+)-Reticuline & O. lancifolia & Fournet et al., 2007 \\
\hline 71 & Tryptophol-5- $O-\beta-\mathrm{D}$-glucopyranoside & O. minarum & Garcez et al., 2005 \\
\hline NMS & Lequesnamine & O. leucoxylon & Imler et al., 2003 \\
\hline 72 & (+)-6S-Dicentrine $N$-oxide & O. acutifolia & Garcez et al., 2011 \\
\hline 73 & (+)-Oxocylonine & O. acutifolia & Garcez et al., 2011 \\
\hline 74 & $(+)-O$-Methylcassyfiline & O. acutifolia & Garcez et al., 2011 \\
\hline 75 & $(+)$-Nordicentrine & O. acutifolia & Garcez et al., 2011 \\
\hline 76 & (+)-Thalicsimidine & O. acutifolia & Garcez et al., 2011 \\
\hline 77 & (+)-Isodomesticine & O. acutifolia & Garcez et al., 2011 \\
\hline 78 & (+)-Predicentrine & O. acutifolia & Garcez et al., 2011 \\
\hline \multirow[t]{2}{*}{79} & (+)-N-Methyllaurotethanine & O. acutifolia & Garcez et al., 2011 \\
\hline & Flavonoids & & \\
\hline 80 & A-type proanthocyanidin trimer & O. notata & Garett et al., 2012 \\
\hline \multirow[t]{2}{*}{81} & Isoquercitrin & O. notata & Garett et al., 2012 \\
\hline & & O. corymbosa & Batista et al., 2010 \\
\hline \multirow[t]{2}{*}{82} & Reynoutrin & O. notata & Garett et al., 2012 \\
\hline & & O. corymbosa & Batista et al., 2010 \\
\hline 83 & Miquelianin & O. notata & Garett et al., 2012 \\
\hline 84 & Quercitrin & O. notata & Garett et al., 2012 \\
\hline 85 & Afzelin & O. notata & Garett et al., 2012 \\
\hline 86 & Catechin & O. notata & Garett et al., 2012 \\
\hline 87 & Epicatechin & O. notata & Garett et al., 2012 \\
\hline 88 & Quercetin & O. notata & Garett et al., 2012 \\
\hline 89 & Kaempferol & O. acutifolia & Garcez et al., 2011 \\
\hline 90 & Quercetin-3-O- $\beta$-D-galactoside & O. corymbosa & Batista et al., 2010 \\
\hline 91 & rel- $(2 R, 3 R)$-dihydroquercetin-3-O- $\alpha$-L-rhamnoside (astilbin) & O. elegans & Batista et al., 2010 \\
\hline 92 & Taxifolin & O. minarum & Garcez et al., 2005 \\
\hline 93 & Quercetin-7-O- $\beta$-D-glucopyranoside & O. minarum & Garcezet al., 2005 \\
\hline 94 & Eriodictyol-3'-O- $\beta$-D-glucopyranoside & O. minarum & Garcez et al., 2005 \\
\hline \multirow[t]{2}{*}{95} & Naringenin-7-O- $\beta$-D-glucopyranoside & O. minarum & Garcez et al., 2005 \\
\hline & Sesquiterpenes & & \\
\hline 96 & rel-4 $4,5 \beta, 7 \beta$-eremophil-9-en-12-oic acid & O. lancifolia & Camargo et al., 2013 \\
\hline 97 & rel-4 $\beta, 5 \beta, 7 \beta$-eremophil-1 (10)-en-12-oic acid & O. lancifolia & Camargo et al., 2013 \\
\hline 98 & 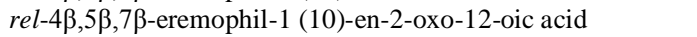 & O. lancifolia & Camargo et al., 2013 \\
\hline 99 & 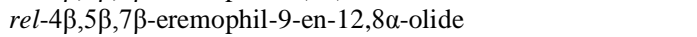 & O. lancifolia & Camargo et al., 2013 \\
\hline 100 & 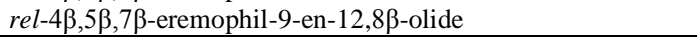 & O. lancifolia & Camargo et al., 2013 \\
\hline
\end{tabular}




\begin{tabular}{|c|c|c|c|}
\hline 101 & 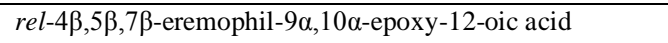 & O. lancifolia & Camargo et al., 2013 \\
\hline 102 & $4 \beta, 5 \beta, 7 \beta$-eremophil-11-en- $10 \alpha$-ol & O. lancifolia & Camargo et al., 2013 \\
\hline 103 & Spathulenol & O. lancifolia & Camargo et al., 2013 \\
\hline 104 & rel- $(1 R, 4 S)$-7-hydroxycalamenene & O. elegans & Batista et al., 2010 \\
\hline 105 & rel- $(8 R)$-Humulan-1,4-dien-8-ol & O. catharinensis & Funasaki et al., 2009 \\
\hline \multirow[t]{2}{*}{106} & Lanceolic acid & O. minarum & Garcez et al., 2005 \\
\hline & Lignans & & \\
\hline 107 & meso-3,4,5,3',4',5'-Hexamethoxy-8.8'-lignan & O. macrophylla & Coy-Barerra et al., 2011 \\
\hline 108 & threo-3,4,5,3',4',5'-Hexamethoxy-8.8'-lignan & O. macrophylla & Coy-Barerra et al., 2011 \\
\hline 109 & erythro-Diarylbutane & O. macrophylla & Suárez et al., 2011 \\
\hline 110 & threo-Diarylbutane & O. macrophylla & Suárez et al., 2011 \\
\hline \multirow[t]{2}{*}{111} & Yangambin & O. heterochroma & Cuca et al., 2009 \\
\hline & & O. duckei & Neto et al., 2007; 2008 \\
\hline \multirow[t]{2}{*}{112} & Lyonyresinol & O. minarum & Garcez et al., 2005 \\
\hline & Butanolides & & \\
\hline 113 & Macrocarpolide A & O. macrocarpa & Liu et al., 2015 \\
\hline 114 & Macrocarpolides B & O. macrocarpa & Liu et al., 2015 \\
\hline 115 & Macrocarpolides C & O. macrocarpa & Liu et al., 2015 \\
\hline 116 & Linderanolide B & O. macrocarpa & Liu et al., 2015 \\
\hline \multirow[t]{2}{*}{117} & Isolinderanolide & O. macrocarpa & Liu et al., 2015 \\
\hline & Benzopyrans & & \\
\hline 118 & 2-Methyl-5-methoxy-benzopyran-4-one & O. corymbosa & Teles et al., 2005 \\
\hline 119 & (2'S)-2-(propan-2'-ol)-5-hydroxy-benzopyran-4-one & O. corymbosa & Teles et al., 2005 \\
\hline 120 & $(2 R)$-2,3-dihydro-2-methyl-5-methoxy-benzopyran-4-one & O. corymbosa & Teles et al., 2005 \\
\hline \multirow[t]{2}{*}{121} & 2,3-Dihydro-2-methyl-benzopyran-4,5-diol & O. corymbosa & Teles et al., 2005 \\
\hline & Steroids & & \\
\hline 122 & $\beta$-Friedelanol & O. heterochroma & Cuca et al., 2009 \\
\hline 123 & 3-O- $\beta$-D-glucopyranosyl stigmasterol & O. minarum & Garcez et al., 2005 \\
\hline 124 & Stigmasta-4,22-dien-3-one & O. minarum & Garcez et al., 2005 \\
\hline \multirow[t]{2}{*}{125} & $\beta$-Sitosterol & O. minarum & Garcez et al., 2005 \\
\hline & Alkylphenols & & \\
\hline 126 & 3- (1,4-Dihydroxypentyl)-5-methoxyphenol & O. minarum & Garcez et al., 2005 \\
\hline \multirow[t]{2}{*}{127} & 5-Propylresorcinol & O. minarum & Garcez et al., 2005 \\
\hline & Arylpropene & & \\
\hline \multirow[t]{2}{*}{128} & trans-Asarone & O. minarum & Garcez et al., 2000 \\
\hline & Coumarin & & \\
\hline \multirow[t]{2}{*}{129} & Scopoletin & O. minarum & Garcez et al., 2005 \\
\hline & Ester & & \\
\hline \multirow[t]{2}{*}{130} & 4-O-E-caffeoylquinic acid methyl ester & O. corymbosa & Batista et al., 2010 \\
\hline & Saponin & & \\
\hline 131 & Guaianin & O. elegans & Oliveira et al., 2006 \\
\hline
\end{tabular}

NMS - no molecular structure provided. 
Table 2: Chemical components identified in the essential oils of the genus Ocotea (2000-2016).

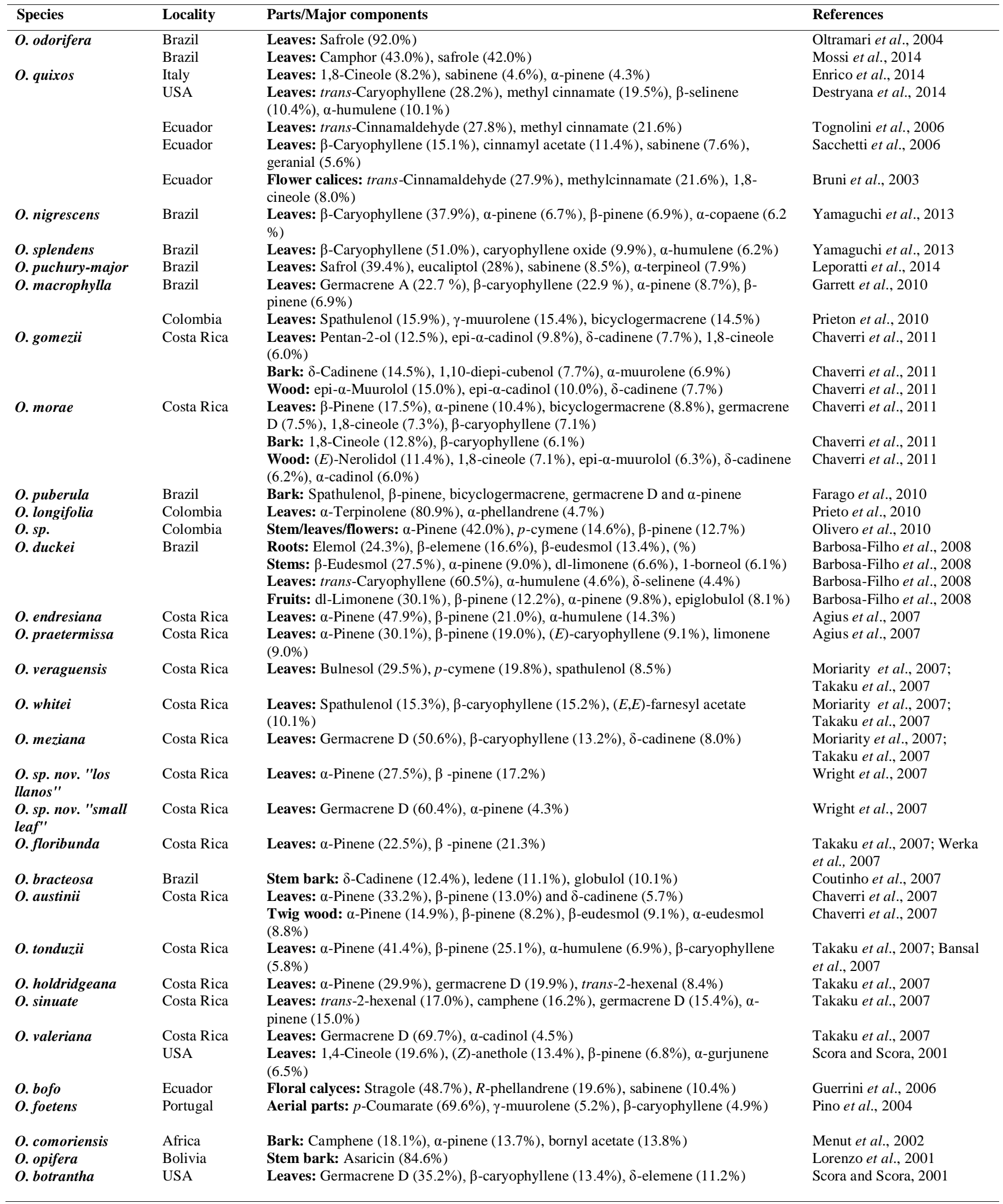




\section{Neolignans}

Forty one neolignans were isolated from eight Ocotea species. Rakotondraibe et al. (2015)successively isolated ten new neolignans including the 6'-oxo-8.1'-lignans, cymosalignan A-C (3-5), 8.O.6'-neolignan (6), ococymosin (7), didymochlaenone C (9), and the bicyclo[3.2.1] loctanoids (11-14) were isolated along with the known compounds, didymochlaenone B (8), 3,4,5,3',5'pentamethoxy-1'-allyl-8.O.4'-neolignan (15), 3,4,5,3'tetramethoxy-1'-allyl-8.O.4'-neolignan (16), virolongin B (17), ocobullenone (18), and sibyllenone (10) from the stems or bark of the Madagascan plant $O$. cymosa. Compound (10) and (18) were also identified from the stem bark of $O$. bullata (Zschocke et al., 2000). Phytochemical exploration of the leaves of $O$. macrophylla afforded two new di-nor-benzofuran neolignan aldehydes, ocophyllals (20-21), and three new macrophyllin-type bicyclo[3.2.1] octanoid neolignans, ocophyllols A-C (22-24). The known compounds of 2'-epi-guianin (19) and licarin B (25) were also isolated (Coy-Barrera et al., 2009; Suarez et al., 2011). Funasaki et al., (2009) reported the isolation of fourteen neolignans (28-41) from the leaves of $O$. catharinensis. They managed to isolate seven new compounds; ( $\left.7 S, 8 S, 1^{\prime} R, 3^{\prime} R\right)$ $3,4,5,3^{\prime}, 5^{\prime}$-pentamethoxy-4'-oxo- $\Delta^{1,3,5,5^{\prime}, 8^{\prime}}-8.1^{\prime}, 7.0 .6^{\prime}$-neolignan (33), rel- $\quad\left(7 R, 8 S, 1^{\prime} R, 2^{\prime} S\right)$-2'-hydroxy-3,4,5'-trimethoxy-3'-oxo$\Delta^{1,3,5,4^{\prime}, 8^{\prime}}-8.1^{\prime}, 7.0 .2^{\prime}$-neolignan (35), rel- $\quad\left(8 S, 1^{\prime} R, 5^{\prime} R\right)-3,4,3^{\prime}, 5^{\prime}-$ tetramethoxy-7, $2^{\prime}$-dioxo- $\Delta^{1,3,5,3^{\prime}, 8^{\prime}}-8.1^{\prime}$-neolignan $\quad(36), \quad r e l-$ ( $\left.7 R, 8 S, 1^{\prime} R, 2^{\prime} S\right)$-2'-hydroxy-3,4-methylenedioxy-5,3',5'trimethoxy- $\Delta^{1,3,5,3^{\prime}, 8^{\prime}}-8.1^{\prime}, 7.0 .2^{\prime}$-neolignan $\quad(37)$, rel$\left(7 S, 8 R, 1^{\prime} R, 3^{\prime} R\right)-4^{\prime}$-hydroxy-3,4-methylenedioxy-3',5'-dimethoxy$2^{\prime}, 4^{\prime}$-dioxo- $\Delta^{1,3,5,5^{\prime}, 8^{\prime}}-8.1^{\prime}, 7.3^{\prime}$-neolignan $\quad(38)$, rel- $\left(7 S, 8 R, 1^{\prime} R, 3^{\prime} R\right)$ 4'-hydroxy-3,4-methylenedioxy-3',5'-dimethoxy-2',4'-dioxo$\Delta^{1,3,5,5^{\prime}, 8^{\prime}}-8.1^{\prime}, 7.3^{\prime}$-neolignan dimer $(\mathbf{4 0})$, and $\left(7 S, 8 R, 1^{\prime} R, 3^{\prime} R\right)-4^{\prime}-$ hydroxy-3,4-methylenedioxy-3',5',5-trimethoxy-2',4'-dioxo$\Delta^{1,3,5,5^{\prime}, 8^{\prime}}-8.1^{\prime}, 7.3^{\prime}$-neolignan dimer (41). In addition, burchellin (1) was isolated from the leaves of $O$. cymbarum (Narciso et al., 2014), while canelin (2) from the stem of O. elegans (Oliveira et al., 2006). Besides, a new bicyclo[3.2.1] octanoid neolignan (26) was isolated from the fruit of $O$. heterochroma, together with a known compound, meso-dehydroguaiaretic acid (27) (Cuca et al., 2009).

\section{Alkaloids}

Most of alkaloids isolated from several Ocotea species were aporphine and benzylisoquinoline alkaloids. Forty one alkaloids were successfully identified from six Ocotea species. Garcez et al. (2005) isolated a new indole alkaloid; tryptophol-5$O$ - $\beta$-D-glucopyranoside (71) from the fruit of $O$. minarum. Six years later, they managed to isolate two new aporphinoid alkaloids; (+)-6S-ocoteine $N$-oxide (44) and norocoxylonine (46) from the leaves and trunk bark of $O$. acutifolia, along with thirteen aporphine analogues (+)-dicentrine (42), (+)-ocoteine (43), (+)leucoxine (45), (+)-thalicminine $(47),(+)$-neolitsine $(48),(S)$ pallidine (68), (+)-reticuline (70), tryptophol-5-O- $\beta$-Dglucopyranoside (71), lequesnamine (NMS), (+)-6S-dicentrine $N$ oxide (72), (+)-oxocylonine (73), (+)-O-methylcassyfiline (74), (+)-nordicentrine (75), and (+)-thalicsimidine (76) and one morphinan alkaloid, $N$-methyllaurotethanine (79) (Garrett et al., 2010). Compound (42) was also isolated from the fruit of $O$. puberula (Montrucchio et al., 2012). Coy-Barrera et al., (2009) reported the isolation of seven aporphine alkaloids from the leaves of O. macrophylla. They were identified as dicentrine (42), neolitsine (48), dehydronantenine (53), nantenine (54), $\mathrm{N}$-acetylnornantenine (55), cassythidine (56), and didehydroocoteine (57). A year later, Pabon and Cuca (2010) managed to isolate four aporphine alkaloids from the wood of $O$. macrophylla, which were (S)-3-methoxy-nordomesticine (49), (S)-N-ethoxycarbonyl-3methoxy-nordomesticine $\quad(\mathbf{5 0}), \quad(S)$ - $N$-formyl-3-methoxynordomesticine (51), and (S)-N-methoxycarbonyl-3-methoxynordomesticine (52). Fournet and co-workers (2007)had successfully isolated thirteen known isoquinoline alkaloids from the stem bark of $O$. lancifolia. It comprises three benzyltetrahydroisoquinolines, coclaurine (58), $N$-methycoclaurine (59), norjuziphine (69), reticuline (70); two pro-aporphines: crostparine (NMS), glaziovine (60); eight aporphines, laurotetanine (62), nordomesticine (63), norisoboldine (64), domesticine (66), isoboldine (67), norantenine (NMS), caaverine (61), corytuberine (65), and the morphidanedienone alkaloid $(S)$-pallidine (68). Compound (58) was also isolated from the stem bark of $O$. duckei (Silva et al., 2002). Besides, Imler et al., (2003)had successfully isolated a new oxoaporphine alkaloid, lequesnamine from the wood of $O$. leucoxylon.

\section{Flavonoids}

Sixteen flavonoids were isolated from five Ocotea species. Garett et al., (2012) have successfully isolated an A-type proanthocyanidin trimer $(\mathbf{8 0})$, isoquercitrin $(\mathbf{8 1})$, reynoutrin $(\mathbf{8 2})$, miquelianin (83), quercitrin (84), afzelin (85), and four minor compounds; catechin (86), epicatechin (87), quercetin (88), and kaempferol $(\mathbf{8 9})$ from the leaves of $O$. notata. They also managed to isolate compound (89) from the leaves of $O$. acutifolia (Garcez et al., 2011). Compounds (81-82) were also isolated from the leaves of $O$. corymbosa, together with quercetin-3-O- $\beta$-Dgalactoside (90) (Batista et al., 2010). In addition, Batista et al., (2010) also isolated a flavonoid, rel- (2R,3R)-dihydroquercetin-3$O$ - $\alpha$-L-rhamnoside (91) from the leaves of $O$. elegans. Garcez et al., (2005) obtained four flavonoids which were taxifolin (92), quercetin-7- $O$ - $\beta$-D-glucopyranoside (93), eriodictyol-3'- $O-\beta$-Dglucopyranoside (94), and naringenin-7- $O-\beta$-D-glucopyranoside (95) from the fruit of $O$. minarum.

\section{Sesquiterpenes}

Eleven sesquiterpenes were isolated from four species of Ocotea. Camargo et al., (2013) isolated six new eremophilane

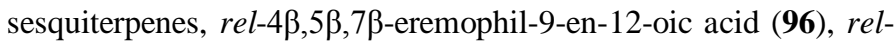

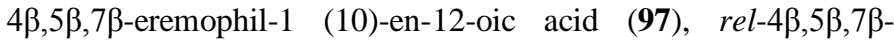

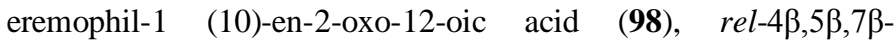
eremophil-9-en-12,8 $\alpha$-olide (99), rel-4 $\beta, 5 \beta, 7 \beta$-eremophil-9-en-12,

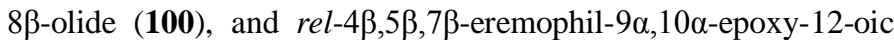
acid (101), from the leaves of $O$. lancifolia, together with two 
known sesquiterpenes, $4 \beta, 5 \beta, 7 \beta$-eremophil-11-en-10 $\alpha$-ol (102) reported fors the first time in the genus Ocotea, and the aromadendrene sesquiterpene, spathulenol $(\mathbf{1 0 3})$. Besides, the sesquiterpene rel- $(1 R, 4 S)$-7-hydroxycalamenene (104) isolated from the leaves of $O$. elegans, has already been isolated from $O$. corymbosa. However, there was no report regarding this compound on other Lauraceae genera (Batista et al., 2010). Additionally, a new sesquiterpene of humulane type, rel- $(8 R)$ humulan-1,4-dien-8-ol (105), besides known spathulenol (103) was isolated form the leaves of $O$. catharinensis (Suarez et al., 2011), while lanceolic acid (106) was identified from the trunk bark of O. minarum (Garcez et al., 2005).

\section{Lignans}

Six lignans were isolated from four species of Ocotea. Two known lignans were isolated from the leaves of $O$. macrophylla which were meso-3,4,5,3',4',5'-hexamethoxy-8.8'lignan (107) and threo-3,4,5,3',4',5'-hexamethoxy-8.8'-lignan (108) (Coy-Barrera et al., 2011). Meanwhile, Suarez et al., (2011)also managed to isolateerythro-diarylbutane (109) and threo-diarylbutane (110) from the same species. Yangambin (111), a furofuran lignan was obtained from the leaves of $O$. duckei (Neto et al., 2007, 2008) and fruits of O. heterochroma (Cuca et al., 2009). Besides, lyonyresinol (112) was isolated from the heartwood of O. minarum (Garcez et al., 2005).

\section{Butanolides}

Five butanolides were isolated from the root of $O$. macrocarpa. Macrocarpolide A (113), B (114) and C (115) were isolated for the first time from genus Ocotea. Compounds (114115) belong to the class of secobutanolides (Liu et al., 2015).

\section{Benzopyrans}

Four benzopyrans derivatives were isolated from the leaves of $O$. corymbosa. (2'S)-2- (propan-2'-ol)-5-hydroxybenzopyran-4-one (119) and 2,3-dihydro-2-methyl-benzopyran4,5-diol (121) were firstly isolated from the genus Ocotea (Teles et al., 2005).

\section{Steroids}

Three steroids, 3-O- $\beta$-D-glucopyranosyl stigmasterol (123), stigmasta-4,22-dien-3-one (124), and $\beta$-sitosterol (125) were isolated from the heartwood and trunkbark of $O$. minarum (Garcez et al., 2005). $\beta$-Friedelanol (122) was obtained from the fruit of $O$. heretochroma (Cuca et al., 2009).

\section{Alkylphenols}

Two alkylphenols, 3- (1,4-dihydroxypentyl)-5methoxyphenol (126) and 5-propylresorcinol (127) were isolated from the heartwood of O. minarum (Garcez et al., 2005)

\section{Miscellaneous compounds}

The arylpropene, trans-asarone (128), and a coumarin, scopoletin (129) were successfully isolated from the heartwood and fruits of $O$. minarum (Garcez et al., 2005). An ester, 4-O-Ecaffeoylquinic acid methyl ester (130) was obtained from the leaves of $O$. corymbosa (Batista et al., 2010), while a saponin, guaianin (131) was found from the leaves of $O$. elegans (Oliveira et al., 2006).

\section{Essential oils}

Since 2000 to 2016, thirty one Ocotea species have been reported on their essential oils composition as shown in Table 2. Sesquiterpenoids were found as the major group components in most of the Ocotea essential oils, which are $O$. quixos, $O$. nirescens, $O$. splendens, $O$. macrophylla, $O$. gomezii, O. puberula, $O$. duckei, O. veraguensis, O. whitei, O. meziana, and $O$. bracteosa (Barbosa-Filho et al., 2008; Chaverri et al., 2007; Coutinho et al., 2007; Destryana et al., 2014; Farago et al., 2010; Garrett et al., 2010; Moriarity et al., 2007; Prieto et al., 2010; Sacchetti et al., 2006; Takaku et al., 2007; Wright et al., 2007; Yamaguchi et al., 2013). In addition, monoterpenoids were also detected from the essential oils of $O$. marae, $O$. longifolia, $O$. endresiana, $O$. praetermissa, $O$. floribunda, $O$. austinii, $O$. tonduzii, and $O$. holdridgeana (Agius et al., 2007; Bansal et al., 2007; Chaverri et al., 2011; Chaverri and Ciccio, 2007; Moriarity et al., 2007; Prieto et al., 2010; Takaku et al., 2007; Werka et al., 2007). Meanwhile, phenylpropanoids were also reported as the major group components isolated in $O$. odorifera, O. puchurymajor, and $O$. opifera (Leporatti et al., 2014; Lorenzo et al., 2001; Mossi et al., 2014; Oltramari et al., 2004).

\section{BIOLOGICAL ACTIVITIES}

The traditional medicinal applications of the Ocotea species have inspired many pharmacological investigations. The pharmacological activities were compiled in this section. Nineteen different biological activities have been reported from the extracts, essential oils, and their isolated compounds.

\section{Cytotoxicity activity}

The cytotoxicity of $O$. gomezii and $O$. morae essential oils were tested on cell lines (CCF-STTG1, Hep3B, HepG2, H460, AGS, N-87, SW-620, MCF-7, and VERO), and found that astrocytoma cells were the most resistant. The leaves and bark oils of $O$. gomezii gave the best activity against SW620 (colon) with $\mathrm{LD}_{50}$ of 122 and $94 \mu \mathrm{g} / \mathrm{mL}$, respectively. While, the wood oil showed cytotoxicity activity against HepG2 (liver) with $\mathrm{LD}_{50}$ of 94 $\mu \mathrm{g} / \mathrm{mL}$. In addition, the leaf, bark, and wood oils of $O$. morae showed activity against AGS (stomach) $\left(\mathrm{LD}_{50}\right.$ of $\left.183 \mu \mathrm{g} / \mathrm{mL}\right)$, SW620 (colon) $\left(\mathrm{LD}_{50}\right.$ of $\left.166 \mu \mathrm{g} / \mathrm{mL}\right)$, and HepG2 (liver) $\left(\mathrm{LD}_{50}\right.$ of $183 \mu \mathrm{g} / \mathrm{mL}$ ), respectively (Chaverri et al., 2011). O. praetermissa and $O$. endresiana leaf oils were notably cytotoxic on MCF-7 cells (with $>97 \%$ killing at $100 \mu \mathrm{g} / \mathrm{mL}$ ) (Agius et al., 2007). O. whitei oil showed activity against M-14 melanoma cells (with 65\% killing at $100 \mu \mathrm{g} / \mathrm{mL}$ ). In addition, $O$. veraguensis oil was active against the estrogen receptor (ER) negative cell line, MDA-MB 231 (with 93\% killing at $100 \mu \mathrm{g} / \mathrm{mL}$ ) (Moriarity et al., 2007). $O$. 
meziana, Ocotea sp. nov. 'los llanos', and Ocotea sp. nov. 'small leaf' have been reported to exhibit in vitro cytotoxic activity on MCF-7 human mammary adenocarcinoma cells (with 100\% killing at $100 \mu \mathrm{g} / \mathrm{mL}$ ) (Wright et al., 2007). O. floribunda oil has showed notable cytotoxic activity $(\geq 50 \%$ killing on at least one cell line) with the best activity against $\mathrm{HepG} 2$ cell (78.8\% killing at $100 \mu \mathrm{g} / \mathrm{mL}$ ) (Werka et al., 2007). The (+)-ocoteine (43) showed the most potent effect against Hep-2 cells and (+)-neolitsine (48) being the most cytotoxic alkaloid to MCF-7 and B16-F10 cells. Thalicminine (47) on the other hand, was the most active compound against 786-0 cells, although it proved only marginally inhibitory to Hep-2 cells and nontoxic to MCF-7 and B16-F10 cells. While, (+)-6S-ocoteine $N$-oxide (44) was found to be only weakly active against Hep-2 and B16-F10 cells ( $\mathrm{IC}_{50}$ of 32.7 and $30.7 \mu \mathrm{g} / \mathrm{mL}$, respectively) and to be devoid of cytotoxicity against MCF-7 and 786-0 cells, as demonstrated by an $\mathrm{IC}_{50}$ value higher than $50 \mu \mathrm{g} / \mathrm{mL}$ in both cases. These results indicated that, compared with (+)-ocoteine (43), $\mathrm{N}$-oxide functionality in (44) reduces its cytotoxic activity (Garcez et al., 2011). Yangambin (111) isolated from $O$. duckei presented cytotoxicity to murine macrophages, measured by the Trypan blue dye exclusion test and MTT reduction assay, resulting in high $\mathrm{IC}_{50}$ values of $187.0 \mu \mathrm{g} / \mathrm{mL}$ and $246.7 \mu \mathrm{g} / \mathrm{mL}$, respectively (Neto et al., 2008).

\section{Antibacterial and antifungal activities}

$O$. praetermissa and $O$. endresiana leaf oils were slightly antibacterial against Bacillus cereus with MIC values of 78 and $156 \mu \mathrm{g} / \mathrm{mL}$, respectively (Agius et al., 2007). O. bofo oil showed fair inhibiting properties against Escherichia coli, Staphylococcus aureus, and Bacillus subtilis with MIC values of $0.16 \mathrm{mg} / \mathrm{mL}$, while a good inhibition against yeast, Yarrowia lypolytica with MIC values of $0.10 \mathrm{mg} / \mathrm{mL}$ (Guerrini et al., 2006). The $O$. quixosoil also showed a dose-dependent antifungal activity against Candida albicans and Saccharomyces cerevisiae (MIC of 0.024 $\mathrm{mg} / \mathrm{ml}$ ), while the antifungal activity against the dermatophyte and phytopathogen strains by the essential oil was relatively good. The growth inhibition percentage against the dermatophyte Trichophyton mentagrophytes was $60 \%$ at the highest concentration tested. However, O. quixosoil performed better against the phytopathogen Pythium ultimum, with an inhibitory action of $85 \%$ at $500 \mathrm{mg} / \mathrm{mL}$ (Bruni et al., 2003). The in vitro antifungal activity of essential oil from $O$. odorifera had weak activity against $C$. albicans and $C$. tropicalis strains involved in oral cavity infections.

A slight antifungal activity was observed with 2.5 $\mathrm{mg} / \mathrm{mL}$ and MIC in 68\% strains (Castro et al., 2011). The essential oil of $O$. longifolia showed significant fumigant activity against Sitophilus zeamais $\left(\mathrm{CL}_{50}\right.$ of $280.5 \mu \mathrm{L} / \mathrm{L}$ ) (Prieto et al., 2010). The alkaloid fraction of $O$. macrophylla was active against Fusarium oxysporum at $250 \mu \mathrm{g} / \mu \mathrm{L}$. The inhibitory activity against the growth of the fungi was moderate at $5 \mu \mathrm{g} / \mu \mathrm{L}$ for $(S)$-3-methoxynordomesticine (49) while the other alkaloids were ineffective, suggesting that the presence of electron withdrawing substituents on nitrogen atom decreased the antifungal activity. In addition, alkaloid (49) also showed antimicrobial activity against Staphylococcus aureus and Enterococcus faecalis with values of $30 \mathrm{AU}$ (Pabon and Cuca, 2010).

\section{Anti-inflammatory activity}

The essential oils of $O$. nigrescens and $O$. splendens have showed a low anti-aggregant factor with $10.8 \%$ and $11.74 \%$, respectively, as compared with the positive standard acetylsalicylic acid which strongly inhibits platelet aggregation $(100 \%)$. The low antiplatelet activity against ADP shown by both essential oils might be due to the absence of phenylpropanoids in their composition (Yamaguchi et al., 2013). O. quixos essential oil significantly reduced LPS-induced NO release from $\mathrm{J} 774$ macrophages at non-toxic concentrations, inhibited LPS-induced COX-2 expression and increased forskolin-induced cAMP production. The essential oil in carrageenan-induced rat paw edema showed anti-inflammatory effect without damaging gastric mucosa (Ballabeni et al., 2010). The diastereomeric lignans, the meso-isomer (107) andthreo-isomer (108) were found to be the potent COX-2/5-LOX dual inhibitor and PAF-antagonist. Compound (107) displayed a lower $\mathrm{IC}_{50}$ value than (108), in contrast to their COX-inhibition. The $\mathrm{IC}_{50}$ value of (107) for 5LOX and PAFwere 46.4 and $38.9 \mu \mathrm{M}$, respectively, while COX-2 for (108) was $15.6 \mu \mathrm{M}$ (Coy-Barrera et al., 2011). 2'-epi-Guianin (19) showed the most potent inhibition of platelet activating factor (PAF)-induced aggregation of rabbit platelets among other neolignans isolated from $O$. macrophyla, with $\mathrm{IC}_{50}$ value $1.6 \mu \mathrm{M}$ (Coy-Barrera et al., 2009). Sibyllenone (10) was the only compound from $O$. bullata which showed good inhibitory activity towards 5-lipoxygenase with $\mathrm{IC}_{50}$ value of $18.6 \mu \mathrm{M}$ (Zschocke et al., 2000).

\section{Antileishmanial activity}

The ethanolic leaves extract of $O$. macrophylla showed the efficacy assay against both Leishmania parasites, $L$. panamensis and L. braziliensis with $\mathrm{EC}_{50}$ values of 98.0 and 85.7 $\mu \mathrm{g} / \mathrm{mL}$,respectively. In addition, erythro-diarylbutane (109) and ocophyllal B (21) the isolated compounds from $O$. macrophylla have shown the best activity against $L$. panamensis $\left(\mathrm{IC}_{50}\right.$ value $26.6 \mu \mathrm{g} / \mathrm{mL}$ ) and $L$. braziliensis $\left(\mathrm{IC}_{50}\right.$ value $36.3 \mu \mathrm{g} / \mathrm{mL}$ ), respectively (Suarez et al., 2011). The crude ethanolic extract, lignoid fraction, and the purified compound, yangambin (111) obtained from $O$. duckei presented antileishmanial activity with $\mathrm{IC}_{50}$ values of $135.7,26.5$, and $49.0 \mu \mathrm{g} / \mathrm{mL}$, respectively on Leishmania chagasi. Meanwhile, for Leishmania amazonensis, the $\mathrm{IC}_{50}$ values were $143.7,48.2$, and $64.9 \mu \mathrm{g} / \mathrm{mL}$ for the crude ethanolic extract, the lignoid fraction, and the purified compound yangambin (111), respectively (Neto et al., 2007).

\section{Cruzain inhibitory activity}

$O$. praetermiss $a$ and $O$. endresiana leaf oils inhibited cruzain with $\mathrm{IC}_{50}$ values of 87.5 and $18.8 \mu \mathrm{g} / \mathrm{mL}$, respectively (Agius et al., 2007). The leaf essential oils of ten species of Ocotea from Costa Rica were examined on the enzyme inhibitory 
activities against cruzain using a fluorometric assay. The $O$. meziana leaf oil was the most active ( $\mathrm{IC}_{50}$ value $14.9 \mu \mathrm{g} / \mathrm{mL}$ ) followed by $O$. whitei $(15.8 \mu \mathrm{g} / \mathrm{mL})$, Ocotea sp. nov. 'los llanos' $(17.1 \mu \mathrm{g} / \mathrm{mL})$, Ocotea $\mathrm{sp}$. nov. 'small leaf' $(19.2 \mu \mathrm{g} / \mathrm{mL})$, and $O$. holdridgeana $(76.9 \mu \mathrm{g} / \mathrm{mL})$. The leaf oils of $O$. floribunda, $O$. tonduzii, and $O$. valeriana were somewhat active ( $\mathrm{IC}_{50}$ value 100$200 \mu \mathrm{g} / \mathrm{mL}$ ), but $O$. sinuata and $O$. veraguensis essential oils were inactive $\left(\mathrm{IC}_{50}>500 \mu \mathrm{g} / \mathrm{mL}\right)$. The cruzain inhibitory activities of the essential oils can be attributed to active sesquiterpenoid components as well as synergistic effects between two or more components (Setzer et al., 2006).

\section{Mutagenic and recombinogenic activities}

The somatic mutation and recombination test (SMART) in wing cells of Drosophila melanogaster was used to test the mutagenic and recombinogenic activities of alkaloids isolated from $O$. acutifolia. Third-stage larvae derived from the standard cross with wing cell markers $m w h$ and/or $\mathrm{flr}^{3}$ were treated chronically. The frequencies of mutant spots observed in marked heterozygous descendants revealed significant dose-dependent genotoxicity for alkaloids; thalicminine (47), (+)-dicentrine (42), (+)-ocoteine (43), and (+)-6S-ocoteine $N$-oxide (44). These alkaloids also induced mitotic recombination. The presence of a methoxyl group at C-3 (as in compound 43) lowers its genotoxic effect relative to that of unsubstituted analogue (42), and the introduction of $\mathrm{N}$-oxide functionality (43 vs. 44) further reduces its genotoxicity. The very planar conformation of oxoaporphine alkaloid (47) may account for its higher genotoxicity vs. its lessplanar analogues (43) and (44) (Guterres et al., 2013).

\section{Antiherpetic activity}

The flavonoid fraction from $O$. notata leaves extract showed antiherpes activity against both herpes simplex viruses;type 1 (HSV-1) and 2 (HSV-2) with $\mathrm{EC}_{50}$ values of 35.8 and $23.5 \mu \mathrm{g} / \mathrm{mL}$, respectively. Moreover, this fraction was more active against HSV-2 than HSV-1. The mechanisms of antiviral action of the flavonoid fraction against the herpesvirus types 1 and 2 were evaluated and the inhibition of different steps of the virus replication cycle was observed. The percentage inhibition obtained for HSV-2 was higher than $90 \%$ in all performed experiments. Differently, for HSV-1, the flavonoid fraction had no effect in pretreatment of cells and showed $60 \%$ of inhibition in virucidal assay (Garett et al., 2012).

\section{Antioxidant activity}

The antioxidant activity (TBARS method) of the essential oil from Ocotea sp., showed the lowest mean effective concentration with $\mathrm{EC}_{50}$ value of $31.1 \mu \mathrm{g} / \mathrm{mL}$ (Olivero et al., 2010). O. bofo oil revealed a remarkable inhibitory scavenging effect on DPPH with inhibition of $64.4 \%$, while in $\beta$-carotene bleaching test gave $75.8 \%$ inhibition. In the photochemiluminescence (PCL) assay, the oil gave $3.14 \mathrm{mmol}$ of Trolox/L (Guerrini et al., 2006). The O. quixos oil exerted a relatively good capacity to act as a non-specific donor of hydrogen atoms or electrons whenchecked by the DPPH assay, quenching $52 \%$ of the radical. On the other hand, it showed weak effects in inhibiting theoxidation of linoleic acid when assayed by the $\beta$ carotene bleaching test (Bruni et al., 2003).

\section{Cardiovascular activity}

The pharmacological activity of $O$. duckei essential oil showing significant cardiovascular effects. The leaves oil induced significant hypotension, followed by intense bradycardia. The fruits also induced a marked hypotension, which was followed by bradycardia. The stem bark and roots were both able to induce hypotension and bradycardia. Among all of the essential oils tested, the hypotensive effect was more potent on diastolic arterial blood pressure compared with the effect induced on systolic pressure (Barbosa-Filho et al., 2008).

\section{Antithrombotic activity}

O. quixos essentially possesses potent and safe antithrombotic activity attributable to its antiplatelet and vasorelaxant effects. The best inhibitory potency against platelet aggregation in guinea pig PRP induced by arachidonic acid $\left(\mathrm{IC}_{50}\right.$ of $47 \mu \mathrm{g} / \mathrm{mL}$ ), while platelet aggregation in human PRP induced by thromboxane $\mathrm{A}_{2}$ receptor agonist $\mathrm{U} 46619\left(\mathrm{IC}_{50}\right.$ of $\left.115 \mu \mathrm{g} / \mathrm{mL}\right)$. The antithrombotic activity could be related to its ability to block both platelet aggregation and clot retraction and to inhibit vasoconstriction (Ballabeni et al., 2007).

\section{Antiproliferative activity}

Macrocarpolides A-C (113-115), linderanolide B (116), and isolinderanolide (117) showed good antiproliferative activities against the $\mathrm{A} 2780$ ovarian cell line, with $\mathrm{IC}_{50}$ values of $2.57,1.98$, $1.67,2.43$, and $1.65 \mu \mathrm{M}$, respectively. The similar $\mathrm{IC}_{50}$ values for the five compounds suggested that they have a similar mechanism of action, possibly as Michael acceptors (Liu et al., 2015). A benzopyran (119) was found to induce cell proliferation: $70 \%$ on $\mathrm{HeLa}$ (human cervix tumour) cells and $25 \%$ on $\mathrm{CHO}$ (Chinese hamster ovary) cells (Teles et al., 2005).

\section{Antinociceptive activity}

Dicentrine (42) produced dose-related inhibition of acetic acid-induced pain without causing changes in the motor performance of mice. Furthermore, the chloroform fraction from $O$. puberula fruit produced marked antinociception in different models of chemical pain, and this effect appears to be, at least in part, due to the presence of dicentrine (42). The mechanisms by the extract and the alkaloid produced antinociception still remains unclear, but the adenosinergic or opioid system seems unlikely to be involved in this action (Montrucchio et al., 2012).

\section{Larvicidal activity}

Burchellin (1) isolated from O. cymbarum interfered with the development cycle of Aedes aegypti, where its strongest toxic effect was $100 \%$ mortality in larvae (L3) at concentrations $\geq 30$ ppm (Narciso et al., 2014). The new 8.O.6'-neolignan (6), 
dihydrobenzofuranoid (7), and bicyclo[3.2.1] octanoid (11) havein vitro activities against Aedes aegypti, with $\geq 80 \%$ mortality at 4 $\mathrm{mg} / \mathrm{mL}$ (Rakotondraibe et al., 2015).

\section{Lethality activity}

$O$. praetermissa and $O$. endresiana leaf oils were active in the brine shrimp lethality test against Artemia salina with $\mathrm{LC}_{50}$ values of 31.6 and $6.9 \mu \mathrm{g} / \mathrm{mL}$, respectively (Agius et al., 2007). The $O$. floribunda oil showed notable brine shrimp toxicity against Artemia salina with $\mathrm{LC}_{50}$ value of $3.7 \mu \mathrm{g} / \mathrm{mL}$. On the other, the oil also showed antibacterial activity on Staphylococcus aureus with MIC value of $78 \mu \mathrm{g} / \mathrm{mL}$ (Werka et al., 2007).

\section{Antiprotozoal activity}

(-)-Caaverine (61) has shown the most interesting antiprotozoal activity against Leishmaniabraziliensis, Leishmania amazonensis, and Leishmania donovani with $\mathrm{IC}_{100}$ of $10 \mu \mathrm{g} / \mathrm{mL}$, respectively. Meanwhile, (-)-caaverine (61) and (+)-domesticine (66) showed the best activity against Trypanosoma cruzi parasite with $\mathrm{IC}_{50}$ of 155 and $105 \mu \mathrm{g} / \mathrm{mL}$, respectively (Fournet et al., 2007).

\section{Toxicity activity}

The essential oil of $O$. notata was evaluated by means of the brine shrimp lethality test and showed a high toxicity with an $\mathrm{LC}_{50}$ value of $2.37 \mu \mathrm{g} / \mathrm{mL}$ (Garrett et al., 2010). The Ocotea sp. essential oil has showed high cytotoxicity $\left(\mathrm{LC}_{50}\right.$ value $\left.7.84 \mu \mathrm{g} / \mathrm{mL}\right)$ against Artemia franciscana on the brine shrimp assay (Olivero et al., 2010).

\section{Molluscicidal activity}

The stem bark oil of $O$. bracteosa showed significant molluscicidal activity against Biomphalaria glabrata, with an $\mathrm{LC}_{90}$ value of $8.3 \mu \mathrm{g} / \mathrm{mL}$, which falls below the threshold of $100 \mu \mathrm{g} / \mathrm{mL}$, set for potential molluscicidal activity by the World Health Organization (Coutinho et al., 2007).

\section{Antiplasmodial activity}

Ococymosin (7) was the most active antiparasitic component than other neolignans, with an $\mathrm{IC}_{50}$ value of $0.45 \mu \mathrm{M}$ against the Dd2 strain of Plasmodium falciparum (Rakotondraibe et al., 2015).

\section{Antilmalarial activity}

The antimalarial activity of the $O$. comoriensis oil is interesting, with an $\mathrm{IC}_{50}$ value of $10 \mu \mathrm{g} / \mathrm{mL}$ (Menut et al., 2002).

\section{CONCLUSION}

The genus Ocotea is widespread all over the world, and many species of this genus have been used as traditional herbal medicines. The chemical investigation of Ocotea species has revealed that many components from this genus exhibit significant bioactivities. Nevertheless, there are still several Ocotea species that have received no or only little attention, and phytochemical and biological studies now should focus on these plants.

\section{Financial support and sponsorship: NIL.}

Conflict of Interests: There are no conflicts of interest.

\section{REFERENCES}

Agius BR, Setzer MC, Stokes SL, Walker TM, Haber WA, Setzer WN. Composition and bioactivity of essential oils of Lauraceae from Monteverde, Costa Rica. Int J Essent Oil Ther, 2007; 1: 167-171

Ballabeni V, Tognolini M, Giorgio C, Bertoni S, Bruni R, Barocelli E.Ocotea quixos Lam. essential oil: In vitro and in vivo investigation on its anti-inflammatory properties. Fitoterapia,2010; 81:289-295.

Ballabeni V, Tognolini M, Bertoni S, Bruni R, Guerrini A, Rueda GM, Barocelli E. Antiplatelet and antithrombotic activities of essential oil from wild Ocotea quixos (Lam.) Kosterm. (Lauraceae) calices from Amazonian Ecuador. Pharmacol Res, 2007; 55: 23-30.

Bansal A, Moriarity DM, Takaku S, Setzer WN. Chemical composition and cytotoxic activity of the leaf essential oil of Ocotea tonduzii from Monteverde, Costa Rica. Nat Prod Commun, 2007; 2: 781784.

Barbosa-Filho JM, Cunha RM, Dias CS, Athayde-Filho PF, Silva MS, Leitao da-Cunha EV, Machado MIL, Craveiro AA, Medeiros IA. GC-MS analysis and cardiovascular activity of the essential oil of Ocotea duckei. Rev Bras Farmacogn, 2008; 18: 37-41.

Batista ANL, Batista JMJ, Lopez SN, Furlan M, Cavalheiro AJ, Silva DHS, Bolzani VS, Nunomura SM, Yoshida M. Aromatic compounds from three Brazilian Lauraceae species. Quim Nova, 2010; 33: 321-323.

Bruni R, Medici A, Andreotti E, Fantin C, Muzzoli M, Dehesa M, Romagnoli C, Sacchetti G. Chemical composition and biological activities of Ishpingo essential oil, a traditional Ecuadorian spice from Ocotea quixos (Lam.) Kosterm. (Lauraceae) flower calices. Food Chem, 2003; 85: 415-421.

Camargo MJ, Miranda MLD, Kagamida CM, Rodrigues ED, Garcez FR, Garcez WS. Sesquiterpenes of Ocotea lancifolia (Lauraceae). Quim Nova, 2013; 36: 1008-1013.

Castro RD, Lima EO. Antifungal activity of Brazilian sassafras (Ocotea odorifera Vell.) and rosemary (Rosmarinus officinalis L.) essential oils against genus Candida. Rev Bras Plant Med, 2011; 13: 203 208.

Coutinho DF, Dias CS, Barbosa-Filho JM, Agra MF, Martins RM, Silva TMS, da-Cunha EVL, Silva MS. Composition and molluscicidal activity of the essential oil from the stem bark of Ocotea bracteosa (Meisn.) Mez. J Essent Oil Res, 2007; 19: 482-484.

Chaverri C, Diaz C, Ciccio JF. Chemical analysis of essential oils from Ocotea gomezii W.C. Burger and Ocotea morae Gomez-Laur. (Lauraceae) collected at "reserva biologica Alberto M. Brenes" in Costa Rica and their cytotoxic activity on tumor cell lines. J Braz Chem Soc, 2011; 22: 741-745.

Chaverri C, Ciccio JF. Essential oils from Ocotea austinii C. K. Allen (Lauraceae) from Costa Rica. J Essent Oil Res, 2007; 19: 439-443.

Christophel DC, Kerrigan R, Rowett AI. The use of cuticular features in the taxonomy of the Lauraceae. Ann Miss Bot Garden, 1996; 83: 419-432.

Coy-Barrera ED, Cuca-Suarez LE. Aporphine alkaloids from leaves of Ocotea macrophylla (Kunth) (Lauraceae) from Colombia. Biochem Syst Ecol, 2009; 37: 522-524.

Coy-Barrera ED, Cuca-Suarez LE, Sefkow M. PAFantagonistic bicyclo[3.2.1]octanoid neolignans from leaves of Ocotea macrophylla Kunth. (Lauraceae). Phytochemistry, 2009; 70: 13091314.

Coy-Barrera ED, Cuca-Suarez LE. In vitro anti-inflammatory effects of naturally-occurring compounds from two Lauraceae plants. Anais Acad Bras Cien, 2011; 83: 1397-1402. 
Coy-Barrera ED, Cuca-Suarez LE, Sefkow M. COX, LOX and platelet aggregation inhibitory properties of Lauraceae neolignans. Bioorg Med Chem Lett,2009; 9:6922-6925.

Cuca LE, Leon P, Coy ED. A bicyclo[3.2.1]octanoid neolignan and toxicity of the ethanol extract from the fruit of Ocotea heterochroma. Chem Nat Comp, 2009; 45: 179-181.

Destryana RA, Young DG, Woolley CL, Huang TC, Wu Hyi, Shih WL.) Antioxidant and anti-inflammation activities of Ocotea, Copaiba and Blue Cypress essential oils in vitro and in vivo. J Am Oil Chem Soc, 2014; 91: 1531-1542.

Enrico R, Matteo M, Silvia M, Gianni S, Renato B. Comparative phytotoxicity of 25 essential oils on pre- and post-emergence development of Solanum lycopersicum L.: A multivariate approach. Ind Crops Prod,2014; 60:280-290.

Farago PV, Padilha-de-Paula JF, Nakashima T, Doll-Boscardin PM, Budel JM, Maia BHLNS. Chemical composition and antibacterial activity of the essential oil from bark of Ocotea puberula (Rich.) Ness. Latin Am J Pharm, 2010; 29: 1242-1245.

Fournet A, Ferreira ME, Rojas de Arias A, Guy I, Guinaudeau $\mathrm{H}$, Heinzen H. Phytochemical and antiprotozoal activity of Ocotea lancifolia. Fitoterapia, 2007; 78: 382-384.

Funasaki M, Lordello ALL, Viana AM, Santa-Catarina C, Floh EIS, Yoshida M, Kato MJ. Neolignans and sesquiterpenes from leaves and embryogenic cultures of Ocotea catharinensis (Lauraceae). J Braz Chem Soc, 2009; 20: 853-859.

Garcez WS, Garcez FR, da Silva LMGE, Shimabukuro AA. Indole alkaloid and other constituents from Ocotea minarum. J Braz Chem Soc, 2005; 16: 1382-1386.

Garcez FR, da Silva AFG, Garcez WS, Linck G, Matos MFC, Santos ECS, Queiroz LMM. Cytotoxic aporphine alkaloids from Ocotea acutifolia. Planta Med, 2011; 77: 383-387.

Garrett R, Cruz RAS, Rocha L, Santos MG, da Silva AJR. Chemical composition and toxicity of Ocotea notata (Nees) Mez essential oil. J Essent Oil Bear Pl, 2010; 13: 455-459.

Garett R, Romanos MTV, Borges RM, Santos MG, Rocha L, da Silva AJR. Antiherpetic activity of a flavonoid fraction from Ocotea notata leaves. Rev Bras Farmacogn, 2012; 22: 306-313.

Guerrini A, Sacchetti G, Muzzoli M, Moreno RG, Medici A, Besco E, Bruni R. Composition of the volatile fraction of Ocotea bofo Kunth (Lauraceae) calyces by GC-MS and NMR fingerprinting and its antimicrobial and antioxidant activity. J Agric Food Chem, 2006; 54: 7778-7788.

Guterres Zda R, da Silva AF, Garcez WS, Garcez FR, Fernandes CA, Garcez FR. Mutagenicity and recombinagenicity of Ocotea acutifolia (Lauraceae) aporphinoid alkaloids. Mut Res, 2013; 757: 91-96.

Imler MJ, Menachery MD, Rajaraman R, Cava MP, Freyer AJ, Shi SD. 2003. Lequesnamine: A new oxoaporphine alkaloid from Ocotea leucoxylon. Abstracts of Papers, 225 ${ }^{\text {th }}$ ACS National Meeting, New Orleans, LA, United States.

Leporatti ML, Pintore G, Foddai M, Chessa M, Piana A, Petretto GL, Masia MD, Mangano G, Nicoletti M. Chemical, biological, morphoanatomical and antimicrobial study of Ocotea puchury-major Mart. Nat Prod Res, 2014; 28: 294-300.

Liu Y, Cheng E, Rakotondraibe LH, Brodie PJ, Applequist W, Randrianaivo R, Rakotondrafara A, Ratsimbason M, Rasamison VE, Kingston DG. Antiproliferative compounds from Ocotea macrocarpa from the Madagascar dry forest. Tetrahedron Lett, 2015; 56: 3630-3632

Lorenzo D, Loayza I, Leigue L, Frizzo C, Dellacassa E, Moyna P. Asaricin, the main component of Ocotea opifera Mart. essential oil. Nat Prod Lett, 2001; 15: 163-170.

Menut C, Bessiere JM, Hassani MS, Buchbauer G, Schopper B. Chemical and biological studies of Ocotea comoriensis bark essential oil. Flav Fragr J, 2002; 17: 459-461.

Montrucchio DP, Miquel OG, Zanin SMW, Araujo da Silva G, Cardozo AM, Santos ARS. Antinociceptive effects of a chloroform extract and the alkaloid dicentrine isolated from fruits of Ocotea puberula. Planta Med, 2012; 78: 1543-1548.
Moriarity DM, Bansal A, Cole RA, Takaku S, Haber WA, Setzer WN. Selective cytotoxic activities of leaf essential oils from Monteverde, Costa Rica. Nat Prod Commun, 2007; 2: 1263-1268.

Mossi AJ, Zanella CA, Kubiak G, Lerin LA, Cansian RL, Frandoloso FS, Prá VD, Mazutti MA, Costa JAV, Treichel H. Essential oil of Ocotea odorifera: An alternative against Sitophilus zeamais. Renew Agric Food Syst, 2014; 29: 161-166.

Narciso JO, Soares RO, Reis dos Santos Mallet J, Guimarães AÉ, de Oliveira Chaves MC, Barbosa-Filho JM, Maleck M Burchellin: study of bioactivity against Aedes aegypti. Parasites Vect, 2014; 7: 172.

Neto RLM, Sousa LMA, Dias CS, Barbosa Filho JM, Oliveira MR. Yangambin cytotoxicity: A pharmacologically active lignan obtained from Ocotea duckei Vattimo (Lauraceae). Z Naturforsch C: J Biosci, 2008 63: 681-686.

Neto RLM, Barbosa-Filho JM, Sousa LMA, Athayde Filho PF, Dias CS, Oliveira MR. Crude ethanolic extract, lignoid fraction and yangambin from Ocotea duckei (Lauraceae) show antileishmanial activity. Z Naturforsch C: J Biosci, 2007; 62: 348-352.

Oliveira R, Heringer A, Figueiredo M, Futuro D, Kaplan M. Isolation of neolignans from Ocotea elegans by CCC. J Liq Chromatogr RT, 2006; 29: 229-234.

Olivero VJ, Gonzalez-Cervera T, Guette-Fernandez J, Jaramillo-Colorado B, Stashenko E. Chemical composition and antioxidant activity of essential oils isolated from Colombian plants. Rev Bras Farmacogn, 2010; 20: 568-574.

Oltramari AC, Wood KV, Bonham C, Verpoorte R, Caro MSB, Viana AM, Pedrotti EL, Maraschin RP, Maraschin M. Safrole analysis by GC-MS of prototrophic (Ocotea odorifera (Vell.) Rohwer) cell cultures. Plant Cell Tiss Org Cult, 2004; 78: 231-235.

Pabon LC, Cuca LE. Aporphine alkaloids from Ocotea macrophylla (Lauraceae). Quim Nova, 2010; 33: 875-879.

Pino JA, Fernandes P, Marbot R, Sa Fontinha S. Chemical composition of the leaf oil of Ocotea foetens (Alt.) Benth. et Hook. from Madeira. J Essent Oil Res, 2004; 16: 131-132.

Prieto JA, Pabon LC, Patino OJ, Delgado WA, Cuca LE. Chemical constituents and insecticidal and antifungal activities of the essential oils of leaves of two Colombian species of the genus Ocotea (Lauraceae). Rev Colomb Quim, 2010; 39: 199-209.

Rakotondraibe LH, Graupner PR, Xiong Q, Olson M, Wiley JD, Krai P, Brodie PJ, Callmander MW, Rakotobe E, Ratovoson F, Rasamison VE, Cassera MB, Hahn DR, Kingston DG, Fotso S. Neolignans and other metabolites from Ocotea cymosa from the Madagascar Rain Forest and their biological activities. J Nat Prod, 2015; 78: 431-440.

Rohwer JG. Toward a phylogenetic classification of the Lauraceae: evidence from matK sequences. Syst Bot, 2000; 60-71.

Sacchetti G, Guerrini A, Noriega P, Bianchi A, Bruni R. Essential oil of wild Ocotea quixos (Lam.) Kosterm. (Lauraceae) leaves from Amazonian Ecuador. Flav Frag J, 2006; 21: 674-676.

Scora RW, Scora PE. Essential leaf oil of Persea subgenus Eriodaphne and closely related Perseoid genera. J Essent Oil Res, 2001; 13: $37-42$.

Setzer WN, Takaku S, Stokes SL, Penton AF. Inhibition of cruzain by Ocotea leaf essential oils from Monteverde, Costa Rica. Pharmacologyonline, 2006; 3: 785-793.

Silva IG, Barbosa-Filho JM, Silva MS, Lacerda CDG, Cunha EVL. Coclaurine from Ocotea duckei. Biochem Syst Ecol, 2002; 30: 881883.

Suarez SJ, Coy-Barrera ED; Cuca LE, Delgado G. Leishmanicidal and cytotoxic activities of extracts and naturally-occurring compounds from two Lauraceae species. Nat Prod Commun, 2011; 6: 231 234.

Takaku S, Haber WA, Setzer WN. Leaf essential oil composition of 10 species of Ocotea (Lauraceae) from Monteverde, Costa Rica. Biochem Syst Ecol, 2007; 35: 525-532.

Teles HL, Silva GH, Castro-Gamboa I, Bolzani VS, Pereira JO, Costa-Neto CM, Haddad R, Eberlin MN, Young MCM, Araujo AR. 
Benzopyrans from Curvularia sp., an endophytic fungus associated with Ocotea corymbosa (Lauraceae). Phytochemistry, 2005; 66: 2363-2367.

Tognolini M, Barocelli E, Ballabeni V, Bruni R, Bianchi A, Chiavarini M, Impicciatore M. Comparative screening of plant essential oils: Phenylpropanoid moiety as basic core for antiplatelet activity. Life Sci,2006; 78:1419-1432.

van der Werff $\mathrm{H}$. A revision of the genus Ocotea Aubl. (Lauraceae) in Madagascar and the Comoro Islands. Adansonia, 2013; 35: 235-279.

Werka JS, Boehme AK, Setzer WN. Biological activities of essential oils from Monteverde, Costa Rica. Nat Prod Commun, 2007; 2: 1215-1219.

Wright BS, Bansal A, Moriarity DM, Takaku S, Setzer WN. Cytotoxic leaf essential oils from neotropical Lauraceae: synergistic effects of essential oil components. Nat Prod Commun, 2007; 2: $1241-$ 1244.

Yamaguchi KKL, Alcantara JM, Lima ES, Veiga-Junior VF. Chemical composition and platelet aggregation activity of essential oils of two species of the genus Ocotea (Lauraceae). J Essent Oil Bear Pl, 2013; 16: 518-523.

Zschocke S, van Staden J, Paulus K, Bauer R, Horn MM, Munro OQ, Brown NJ, Drewes SE. Stereostructure and anti-inflammatory activity of three diastereomers of ocobullenone from Ocotea bullata. Phytochemistry, 2000; 54: 591-595.

\section{How to cite this article:}

Salleh WMN, Ahmad F. Phytochemistry and Biological Activities of the Genus Ocotea (Lauraceae): A Review on Recent Research Results (2000-2016). J App Pharm Sci, 2017; 7 (05): 204-218. 